THE AstrophySICAL JouRnaL, 286:609-629, 1984 November 15

(C) 1984. The American Astronomical Society. All rights reserved. Printed in U.S.A.

\title{
SPECTROPHOTOMETRY OF COMPACT EMBEDDED INFRARED SOURCES IN THE 0.6-1.0 MICRON WAVELENGTH REGION
}

\author{
P. J. McGregor ${ }^{1}$ AND S. E. Persson ${ }^{1}$ \\ Mount Wilson and Las Campanas Observatories of the Carnegie Institution of Washington \\ AND \\ J. G. COHEN \\ California Institute of Technology \\ Received 1983 November 28 ; accepted 1984 June 4
}

\begin{abstract}
Spectrophotometry at $20 \AA$ resolution in the $0.6-1.0 \mu \mathrm{m}$ wavelength range is presented for the compact embedded infrared sources GL 490, GL 437S, IC 1848A, GL 961, S106/IRS 3, M17/IRS 1, S235B, and M8E, for the compact $\mathrm{H}$ II regions GL $437 \mathrm{~W}$ and NGC 7538/IRS 2, and for the visible objects LkH $\alpha$ 101, MWC 297, MWC 349, V645 Cyg, and the fan nebula associated with GL 989. The heavily obscured objects display extremely red continua, emission lines due to the Paschen series of hydrogen, and in many cases O I $\lambda 8446$ and the Ca II infrared triplet lines at $\lambda 28498,8542$, and 8662 , plus other weaker lines. These and other newly identified spectral characteristics of the embedded sources show similarities to those of Be stars, T Tauri stars, and to a class of emission-line stars with infrared excesses. These facts point to similar physical conditions in the circumstellar envelopes of the compact embedded sources and those of other young stellar objects that have long histories of study.

Estimates of the source extinctions have been obtained from the continuum slopes in this region. Comparison of the $1 \mu \mathrm{m}$ continuum magnitudes with estimates for the stellar photospheric fluxes based on the source luminosities suggest that scattering and circumstellar shell emission are important in forming the $1 \mu \mathrm{m}$ continuum in several sources. The Paschen line ratios are not in general consistent with Case B recombination; this extends a conclusion deduced from the ratios of the infrared hydrogen lines $\mathrm{Br} \alpha, \mathrm{Br} \gamma$, and $\mathrm{Pf} \gamma$. The Paschen line decrements are qualitatively consistent with high optical depth in these lines; restrictions on possible excitation mechanisms and geometries are deduced. In those cases where they are detected, the $\mathrm{Ca}$ II triplet lines are equal in strength. This indicates that the optical depths in these lines are substantial. The $\mathrm{O}$ I 28446 line is excited by Ly $\beta$ fluorescence in most of the objects. This shows that the $\mathrm{H}$ I lines are excited in predominantly neutral regions and that the $\mathrm{H} \alpha$ optical depth is $\sim 1000$. The physical conditions in the dense, warm, and only partially ionized medium required in the embedded sources may be similar to those proposed for the broadline region of Seyfert galaxies and QSOs, but $T_{e}$ is probably $\sim 5000 \mathrm{~K}$.
\end{abstract}

Subject headings: infrared: sources - nebulae: H II regions - spectrophotometry - stars: emission-line

\section{INTRODUCTION}

The compact, luminous, and heavily reddened infrared sources, of which the BN object in Orion is the prototype, have attracted considerable attention because they were at one time believed to be "protostars," i.e., objects in the process of collapse to stellar configurations. More recent work on the infrared $\mathrm{H}$ I line fluxes and radio continuum fluxes indicates that some may already be bona fide stars surrounded by ultracompact $\mathrm{H}$ II regions, losing mass at high rates and driving bipolar outflows of molecular gas (see, e.g., Bally and Lada 1983). The infrared searches and follow-up studies of objects in this heterogeneous class have been reviewed by Wynn-Williams (1982). Königl (1982) has recently given a brief summary of a wide range of observational data and has interpreted these objects and their high-momentum outflows in terms of a young star whose wind is channeled into two oppositely directed flows by a dense surrounding disk or toroid.

With the recent development of CCD detectors and spectrometers, objects such as these can now be studied at "visible" wavelengths, whereas previously they were accessible

\footnotetext{
${ }^{1}$ Observations were made at Palomar Observatory as part of a collaborative agreement between the Carnegie Institution of Washington and the California Institute of Technology.
}

only to infrared photometric and spectroscopic systems operating at wavelengths longer than $1 \mu \mathrm{m}$. This paper reports the results of a survey of compact embedded sources in the spectral region $0.6-1.0 \mu \mathrm{m}$. We discuss CCD spectra at $20 \AA$ resolution of 15 sources which show one or more characteristics of extreme youth. Most appear in the list of Wynn-Williams (1982), but we also include data on NGC 7538/IRS 2, MWC 297, and MWC 349. Although the BN source itself is, unfortunately, too faint to be observed in this wavelength range, several sources in our list are believed to be close analogs of $\mathrm{BN}$, but less heavily reddened.

In carrying out our observations we hoped to shed light on several problems that have arisen in infrared studies of these sources. First, the $\mathrm{Br} \alpha$ and $\mathrm{Br} \gamma$ hydrogen lines appear to be too strong relative to the ionizing photon fluxes inferred from their total luminosities. This "line excess" problem, which is more properly termed a line excitation problem, has been discussed in detail by Thompson $(1981,1982)$ and by Thompson, Thronson, and Campbell (1983). Measurements of the Paschen lines in the $0.82-1.0 \mu \mathrm{m}$ range should help to define this problem more clearly. Second, in some sources the infrared hydrogen line profiles imply gas velocities of several hundred $\mathrm{km} \mathrm{s}^{-1}$. The $\mathrm{Br} \alpha$ and $\mathrm{Br} \gamma$ lines in $\mathrm{BN}$, for example, have full width at zero intensity (FWZI) velocities of $\sim 350 \mathrm{~km} \mathrm{~s}^{-1}$ (Scoville et al. 
1982), with similar values for M17, GL 490 (Simon et al. 1981), and a number of other objects (Persson et al. 1984). It has been suspected that there must be a connection between the line excitation problem, the large velocity widths, and the much larger scale bipolar outflows of molecular gas, but very little information exists on the physical conditions or geometrical distributions of the gas close to the central star. It was hoped that an improved understanding of the hydrogen line emission mechanism would help to clarify this connection. Optically thick wind models such as those of Krolik and Smith (1981) and spherically symmetric outflow models for the line shapes (Simon et al. 1981, 1983) appear capable of producing the line fluxes, but the basic assumptions of these models do not necessarily follow from the available data. Third, the infrared continua of the embedded sources are dominated by dust emission; the embedded stars are not seen. Observations in the $0.6-1.0 \mu \mathrm{m}$ range raise the possibility of detecting the photospheric light of the exciting star and measuring its color.

In assessing the results presented in this paper it is important to keep in mind the heterogeneous nature of the sample of sources. There is certainly a range of age represented, but any evolutionary or generic connections between the sources are obscure, except in the broadest sense. For example, a few of the sources, such as NGC 7538/IRS 2, GL 437W, and S106, have extended radio emission; these must be significantly older than objects such as GL 490. In this paper we present only the spectrophotometric results and a brief discussion. Further observations of many of the sources have been obtained at a resolution of $\sim 1.5 \AA$ at the $\mathrm{H} \alpha$ line and in the $\mathrm{Ca}$ II triplet region, corresponding to velocity resolutions of $\sim 70$ and 50 $\mathrm{km} \mathrm{s}^{-1}$, respectively. These data will be presented in future papers (Persson, McGregor, and Cohen 1984).

\section{OBSERVATIONS}

The observations were made during parts of five nights in 1982 December, 1983 July, and 1983 September with the Double Spectrograph (Oke and Gunn 1982) mounted on the Hale $5 \mathrm{~m}$ telescope. Although both the blue and the red sections of the instrument were used, useful data were obtained only from the red side for the highly reddened objects in our sample. The detector was a Texas Instruments $800 \times 800$ pixel CCD. The spectral resolution of $\sim 20 \AA$ and the 800 pixels along the dispersion give spectral coverage between 0.62 and $1.04 \mu \mathrm{m}$. There are 256 pixels along the slit, each pixel corresponding to 0 ". 6 on the sky. The slit width was set to $4^{\prime \prime}$ for most of the observations to ensure that reasonably accurate spectrophotometry could be obtained. (The slit width was reduced to $2^{\prime \prime}$ for a few observations in order to reduce foreground emission from associated $\mathrm{H}$ II regions.) The spectrophotometric standards BD $+17^{\circ} 4708$, HD 19445, and HD 84937 were observed in the same manner as the objects in order to flux-calibrate the raw spectra. In no case was an object or standard observed at an air mass greater than 1.5 , so differential refraction (which is small over this wavelength range) should not introduce spurious color terms, especially in view of the use of a 4 " slit. The seeing was uniformly good, less than $2^{\prime \prime}$ during most of the sessions, and the final continuum flux densities should be accurate to about $10 \%$, excluding uncertainties in the calibration (see Oke and Gunn 1983).

For each object, the telescope was offset from a positional standard to the position of the object as given by WynnWilliams (1982). The reflecting surfaces of the spectrograph slit assembly were viewed with an integrating Quantex television camera through a red filter in an attempt to identify each object and center it on the slit. Nearly every object in the list was seen in this way. For several objects the instrument was rotated to yield spectra of more than one object along the slit. The Appendix gives details on the observation of some objects for which identification was difficult, or in which other problems were encountered. To summarize the main issue of the Appendix, we are confident that in most cases the spectra obtained are due to the point sources generally accepted as being the infrared objects listed by Wynn-Williams (1982). Three exceptions are IC 1848A and GL 989, for which our spectra are of the nearby fan shaped reflection nebulae, and M8E where our spectrum is of a faint nebulous patch at the position of the infrared source; we could not be sure that the slit crossed the true infrared sources, and they were apparently not detected directly in the $0.6-1.0 \mu \mathrm{m}$ region.

Data reduction consisted of first flattening the twodimensional frames, and then subtracting a sky level established close to each object. The spectrum of the object was then extracted by summing 5 to 10 pixels $\left(3^{\prime \prime}\right.$ to $\left.6^{\prime \prime}\right)$ along the slit. Wavelength calibration was achieved by polynomial fitting to neon arc lines. The rms error in the wavelength fit was less than $3 \AA$. Finally, these summed raw counts were converted to flux densities using the standard star spectra and correcting for air mass using a standard atmospheric transmission function. The flux densities of the standards are on the $\mathrm{AB}_{79}$ system of Oke and Gunn (1983). ${ }^{2}$ During the data reduction, each spectrum was carefully checked (using a television display of the raw two-dimensional spectrum) for line or continuum emission which extended beyond the point source. In several cases extended line emission was strong, and improper allowance for it could seriously distort the line ratios. The Appendix gives details on these and other findings for each object in which the reduction was not completely straightforward.

Figure 1 displays the final reduced spectra. At some wavelengths the total accumulated charge on the CCD was large enough to saturate the readout electronics; these positions and the positions of atmospheric bands where minor problems were encountered in the calibration have been omitted from Figure 1. Emission line strengths were obtained from the spectra by linearly interpolating the underlying continuum in the vicinity of each line; the results are given in Tables 1 and 2 . The weaker lines have large uncertainties which arise from difficulties in placing the continuum level. The spectral resolution is not high enough to ensure that all of the weaker lines in Table 2 have been correctly identified. It is also possible that the Paschen line strengths in Tables 1 and 2 deviate systematically from the true emission line strengths because the underlying stellar photosphere may have the Paschen lines in absorption. The absorption equivalent width of $\mathrm{P} 7$ in the B3 star $\eta \mathrm{UMa}$ is about $1 \AA$, according to the spectrum of Johnson (1978), so correction for P7 absorption would not exceed $\sim 20 \%$ for any object. Corrections for underlying absorption in the other Paschen lines should be of the same order.

\section{RESULTS}

Before discussing the results, we emphasize again that the spectra in Figure 1 do indeed represent the objects originally found in infrared searches. Figure 2 shows the new continuum

\footnotetext{
${ }^{2}$ The standards have the $\mathrm{Ca}$ II and Paschen lines in absorption. Proper account of these narrow features was taken in producing the flux-calibrated spectra.
} 


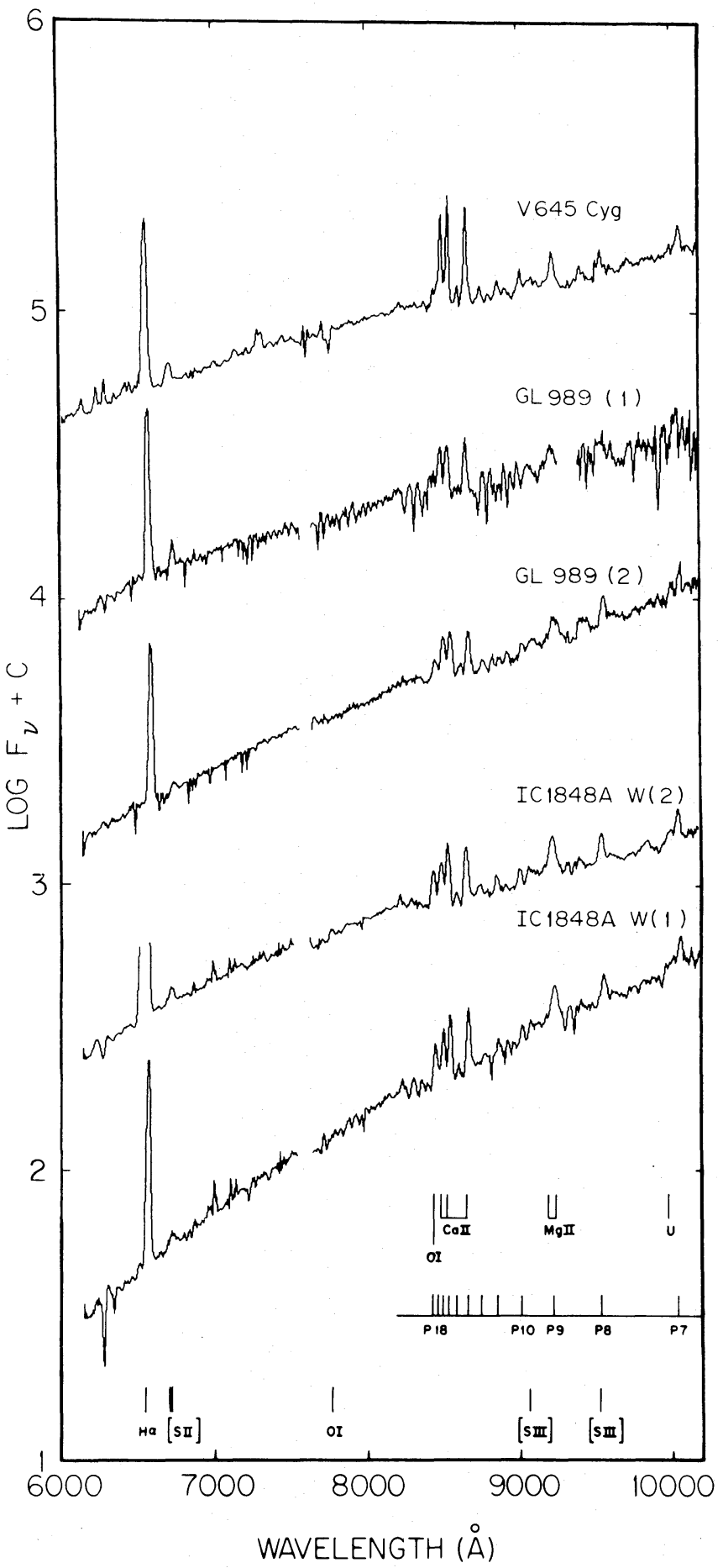

FIG. $1 a$

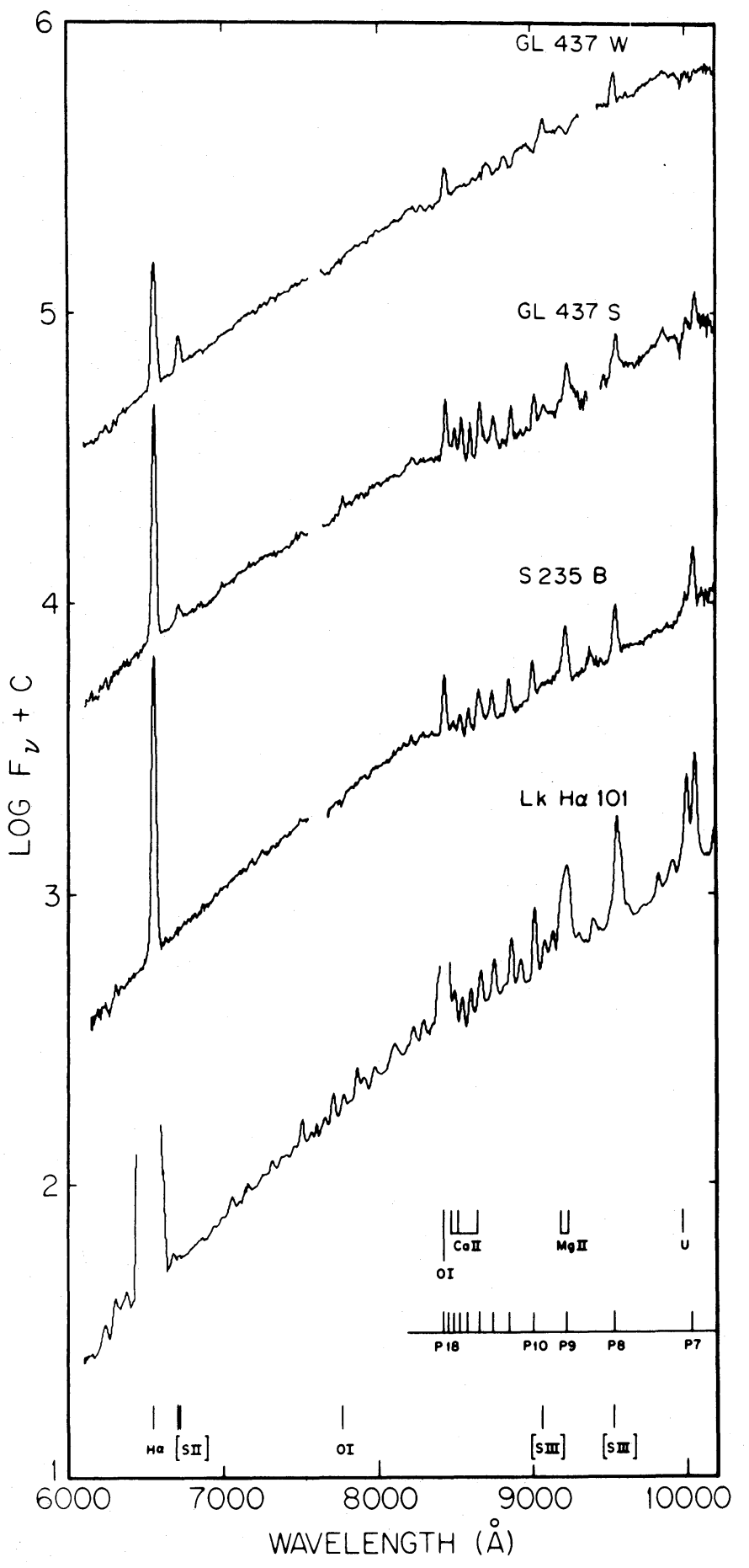

FIG. $1 b$

FIG. 1.-CCD spectra at $20 \AA$ resolution of infrared objects. Several of the important emission lines are identified. The flux density scale is in W $\mathrm{m}^{-2} \mathrm{~Hz}^{-1}$ but has an arbitrary zero-point; the actual continuum flux densities are given in Table 3. Positions where correction for terrestrial atmospheric features was poor or where the CCD detector saturated have been omitted. 


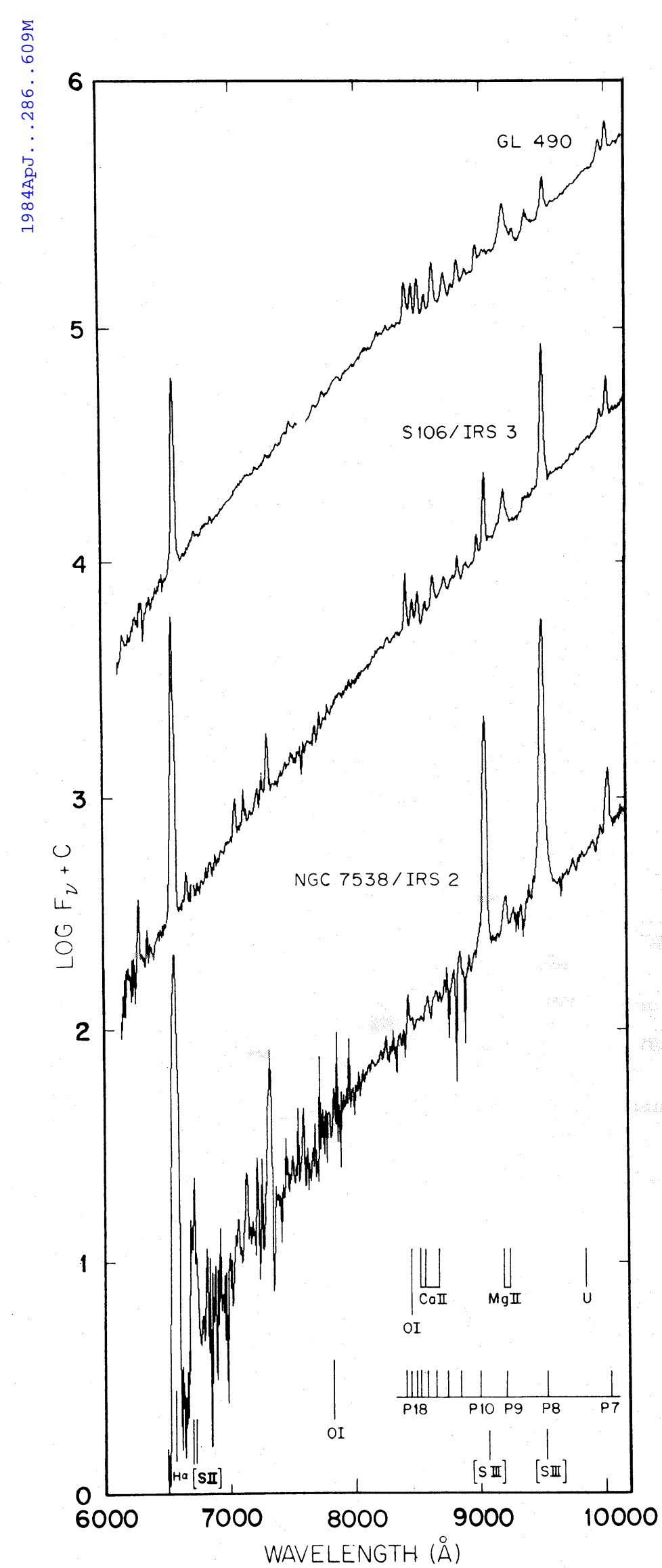

FIG. 1c

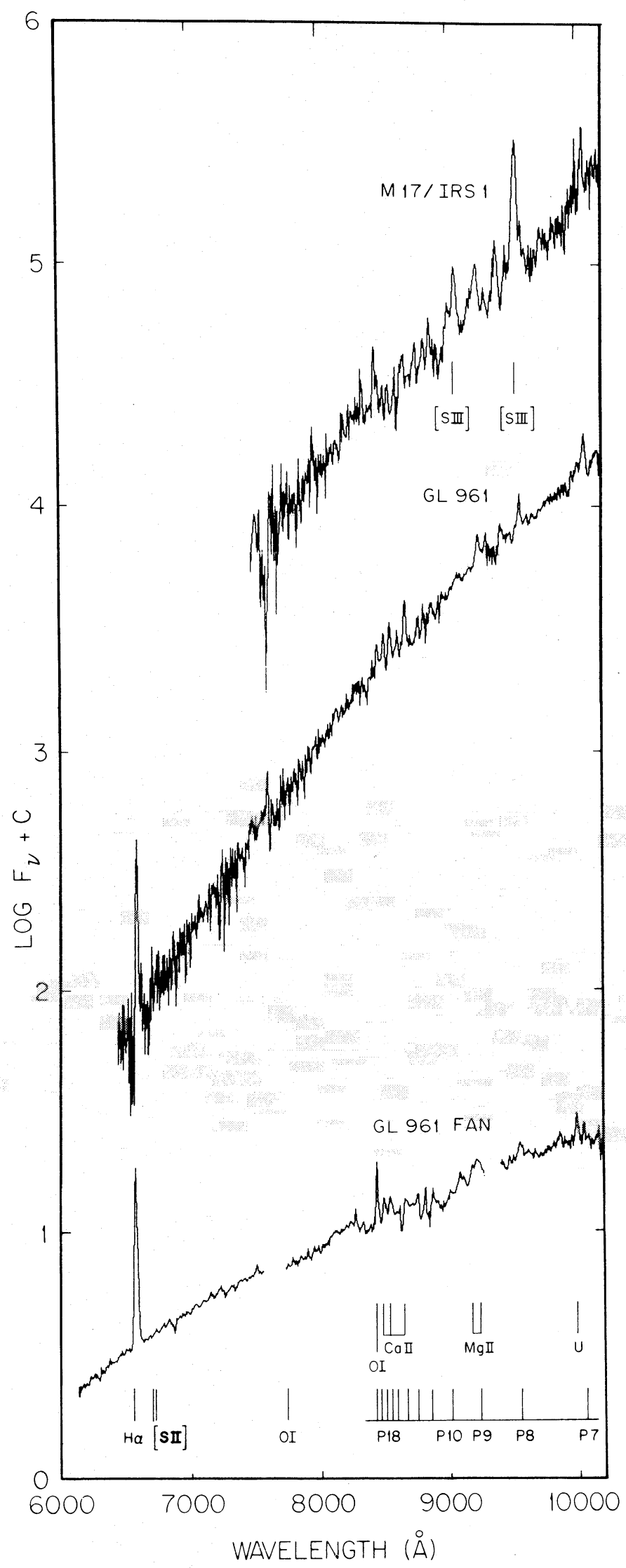

Fig. $1 d$ 


(1)

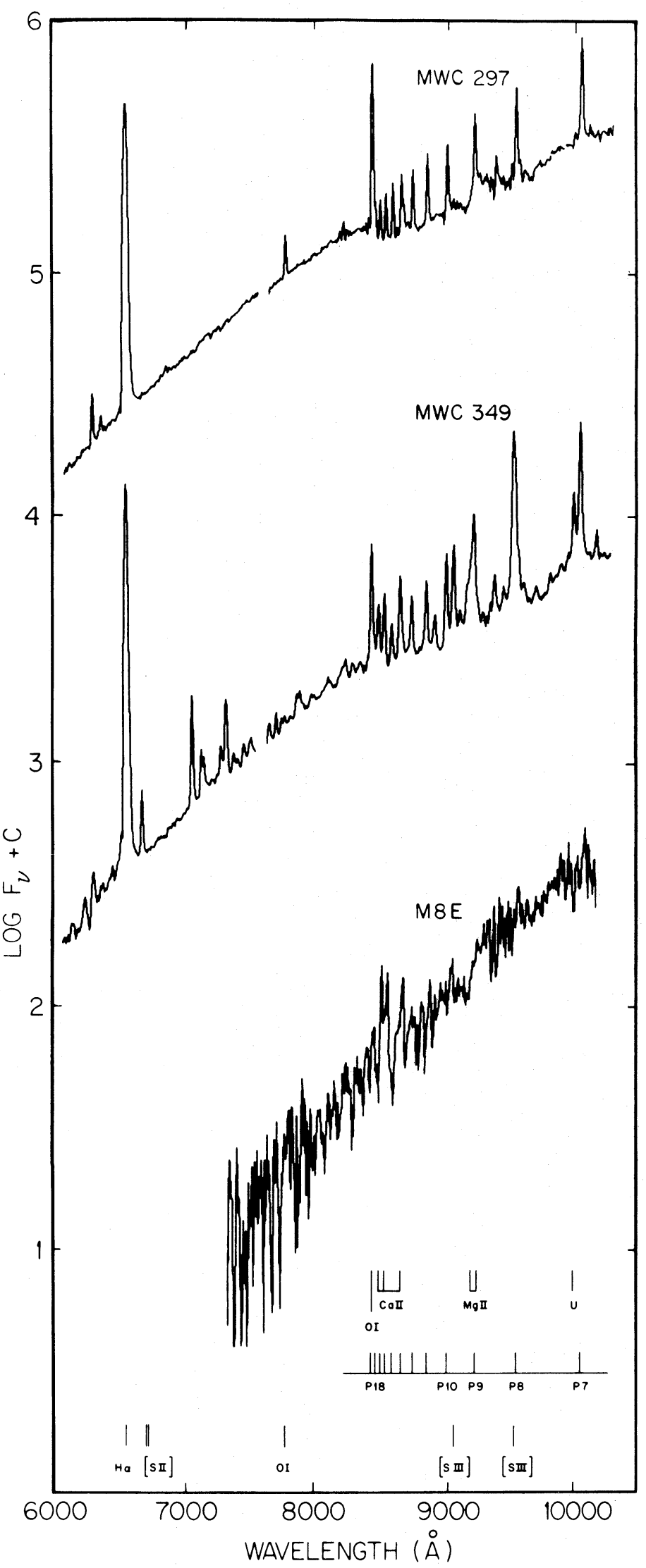

FIG. $1 e$ data plotted together with broad band $1.25,1.65,2.2$, and 3.5 $\mu \mathrm{m}$ photometry taken from the literature (see Table 7). Continuum flux densities in the $0.6-1.0 \mu \mathrm{m}$ range for the spectra shown in Figure 1 are listed in Table 3. Despite the difficulty of joining the separate pieces of the extremely steep energy distributions, they appear to tie together well for most of the objects. This confirms that the visually identified objects and the infrared sources are intimately associated. The exceptions are IC 1848A, GL 989, and M8E. In each of these cases the disagreement is not surprising in view of the spatial distributions of the emission or observational difficulties as discussed in the Appendix. The dashed curves also shown in Figure 2 are discussed below.

\section{a) Major Features of the Spectra}

The spectra plotted in Figure 1 show a number of strong emission lines. The major lines are the Paschen series of $\mathrm{H} \mathrm{I}$, the 28446 line of $\mathrm{O}$ I, and the Ca II infrared triplet lines at $\lambda \lambda 8498,8542,8662$. An emission feature near $9994 \AA$ is also present in many of the spectra. The [S III] $\lambda \lambda 9069,9532$ lines are very strong in NGC 7538/IRS 2 . The continua of several objects show evidence for a weak Paschen jump in emission.

The O I $\lambda 8446$ line is present in most of the objects, and is especially strong in the spectra of $\mathrm{LkH} \alpha 101, \mathrm{~S} 235 \mathrm{~B}, \mathrm{GL} 437 \mathrm{~S}$, GL 437W, S106/IRS 3, MWC 297, and MWC 349. Herbig (1971) reported the spectrum of $\mathrm{LkH} \alpha 101$ in this region to be dominated by $\mathrm{H} \alpha$ and the $\mathrm{O}_{\mathrm{I}}$ line, as we also find. The $\mathrm{O}_{\mathrm{I}}$ 28446 line is known to be strong in a wide variety of objects, including Be stars (e.g., Polidan 1980), T Tauri stars (e.g., Herbig and Soderblom 1980), novae (e.g., Strittmatter et al. 1977), Seyfert galaxy nuclei (Grandi 1980), and QSOs (e.g., Oke and Shields 1976).

The detection of the $\mathrm{Ca}$ II triplet lines at 8498,8542 , and $8662 \AA$, in emission, in most of the young objects is an unexpected result of the observations. The lines are especially strong and obvious in Figure 1 for GL 989 and V645 Cyg, but are measurable in every object except M17/IRS 1, NGC 7538/ IRS 2, GL 437W, and S235B. It is interesting that in the three latter cases there is evidence for radio continuum emission or extended $\mathrm{H}$ I line emission (Wynn-Williams, Becklin, and Neugebauer 1974; Wynn-Williams et al. 1981; the Appendix, respectively), characteristic of compact $\mathrm{H}$ II regions. The $\mathrm{Ca}$ II triplet lines are found in emission in a wide variety of other astrophysical objects. They occur in a majority of the peculiar emission-line stars studied by Ciatti, d'Odorico, and Mammano (1974), Andrillat and Swings (1976), and Swings and Klutz (1976); their occurrence in these objects is apparently related to the amount of excess near-infrared emission (Andrillat and Swings 1976). Herbig and Soderblom (1980) have discussed the occurrence of $\mathrm{Ca}$ II triplet emission in $\mathrm{T}$ Tauri stars and have drawn comparisons between the regions producing $\mathrm{Ca}$ II emission in these stars and in solar flares and plages. The lines have also been seen in nova ejecta, late-type stars, and several other kinds of objects (Polidan 1980), but of particular interest here is their occurrence in about $20 \%$ of the 300 Be stars surveyed by Polidan (1980; see also Polidan and Peters 1976; Polidan 1976). Of all the types of objects displaying $\mathrm{Ca}$ II triplet emission, the Be stars are the only ones with temperatures above $10,000 \mathrm{~K}$. For example, Of and WolfRayet stars do not show Ca II emission. It is believed that in the $\mathrm{Be}$ stars the $\mathrm{Ca}$ II emission originates in a cool circumstellar disk. Ca II triplet emission thus favors a cool environment.

The spectrum of NGC 7538/IRS 2 in Figure 1 shows very 
TABLE 1

EMISSION LINE FLUXES ${ }^{2}$

\begin{tabular}{|c|c|c|c|c|c|c|c|c|c|c|c|c|c|c|c|c|}
\hline Line & ID & $\begin{array}{c}\text { IC } 1848 \mathrm{~A} \\
W(1)\end{array}$ & $\begin{array}{c}\text { IC } 1848 \mathrm{~A} \\
W(2)\end{array}$ & GL $437 \mathrm{~W}$ & GL $437 \mathrm{~S}$ & GL 490 & S235B & GL 961 & $\begin{array}{c}\text { GL } 961 \\
\text { FAN }\end{array}$ & GL $989(1)$ & GL989(2) & M8E & $\mathrm{M} 17 / \mathrm{IRS} 1$ & $\begin{array}{l}\text { S106/3 } \\
\text { IRS 3 }\end{array}$ & $\begin{array}{l}\text { v645 Cyg } \\
\text { (NO) }\end{array}$ & $\begin{array}{c}\text { NGC } 7538 / \\
\text { IRS } 2\end{array}$ \\
\hline (1) & (2) & (3) & (4) & (5) & (6) & (7) & (8) & (9) & (10) & (11) & (12) & (13) & (14) & (15) & (16) & (17) \\
\hline 6563 & $\mathrm{H} \alpha$ & 3.79 & 23.2 & 5.49 & 5.88 & 1.95 & sat. & 0.065 & 6.0 & $\begin{array}{l}4.10 \\
0.22\end{array}$ & 7.82 & $\cdots$ & $\cdots$ & 3.53 & 82.5 & 7.91 \\
\hline $6717+6731$ & {$\left[\begin{array}{ll}S & \mathrm{II}\end{array}\right]$} & 0.19 & 0.86 & 0.99 & 0.31 & & & 10013 & & 0.23 & 0.37 & $\cdots$ & $\cdots$ & & 6.35 & 0.61 \\
\hline 7002 & 0 I & $0.18:$ & 0.56: & $<0.02$ & 0.02: & $<0.004$ & $<0.045$ & $\begin{array}{l}<0.013 \\
<0.0024\end{array}$ & $\begin{array}{l}<0.06 \\
<0.08\end{array}$ & $\ldots$ & $\begin{array}{l}<0.036 \\
<0.045\end{array}$ & $\cdots$ & $\cdots$ & $\begin{array}{l}<0.01 \\
<0.023\end{array}$ & $\ldots$ & $\ldots$ \\
\hline $\begin{array}{l}7774 \\
8446\end{array}$ & $\begin{array}{l}0 \mathrm{I} \\
0 \mathrm{I}+\mathrm{P} 17,18\end{array}$ & $\begin{array}{r}<0.05 \\
3 \quad 0.98\end{array}$ & $\begin{array}{l}0.20: \\
2.87\end{array}$ & $\begin{array}{r}<0.09 \\
2.25\end{array}$ & $\begin{array}{l}0.15: \\
1.71\end{array}$ & $\begin{array}{r}<0.03 \\
1.25\end{array}$ & $\begin{array}{c}<0.26 \\
9.4\end{array}$ & 0.07 : & 1.55 & $\cdots$ & 0.78 & $\cdots$ & $0.07:$ & 1.15 & $\cdots$ & $\cdots$ \\
\hline 8498 & Ca II+P16 & 1.16 & 3.34 & $\ldots$ & $0.70:$ & 0.92 & & $0.13:$ & 0.69 & 0.75 & 1.92 & 0.49 & $\ldots$ & 0.51 & 19.6 & $\ldots$ \\
\hline 8542 & $\mathrm{Ca} \mathrm{II}+\mathrm{P} 15$ & 1.58 & 4.96 & $\ldots$ & 0.88 & 0.96 & 2.37 & $0.14:$ & 0.70 & 0.82 & 2.16 & & $\ldots$ & 0.74 & 23.1 & $\ldots$ \\
\hline 8598 & $\mathrm{P} 14$ & 0.21 & 0.84 & $\ldots$ & 0.56 & 0.50 & 3.00 & $0.74:$ & & & 0.49 & & $\ldots$ & 0.36 & 2.95 & $\cdots$ \\
\hline 8662 & Ca II $+P 13$ & 1.58 & 4.89 & $\ldots$ & 1.62 & 1.51 & 6.64 & 0.23 & 0.20 & 0.76 & 2.14 & 0.29 & $\ldots$ & 0.81 & 22.2 & $\cdots$ \\
\hline 8750 & P12 & wk & 1.20 & abs & 1.05 & 0.91 & 5.52 & wk & $\cdots$ & $\ldots$ & wk & $\cdots$ & wk & 0.55 & 4.29 & $\cdots$ \\
\hline 8863 & P11 & wk & 1.38 & abs & 0.82 & 0.97 & 5.39 & wk & $\ldots$ & $\cdots$ & $\ldots$ & $\cdots$ & wk & 0.73 & 4.42 & $\ldots$ \\
\hline 9015 & P10 & $0.27:$ & 1.04 & abs & 0.84 & 0.98 & 4.38 & wk & $\cdots$ & $\cdots$ & wk & $\cdots$ & wk & 0.73 & 3.57 & \\
\hline 9069 & {$\left[\begin{array}{lll}S & \text { III }\end{array}\right]$} & wk & wk & 1.18 & 0.61 & & & 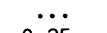 & 1.0 & $\cdots$ & & $\cdots$ & $0.27:$ & 3.60 & & 30.8 \\
\hline $9229+$ & $\mathrm{P} 9+\mathrm{bl}$ end $^{4}$ & 1.50 & 5.02 & abs & 2.51 & 5.12 & 12.3 & $0.25:$ & 2.2 & $\cdots$ & 2.43 & $\cdots$ & $0.45:$ & 3.76 & 14.5 & 1.68 \\
\hline $9532+9546$ & {$\left[\begin{array}{lll}S & I I I\end{array}\right]+P 8$} & $\begin{array}{ll}3 & 0.91\end{array}$ & 2.77 & 3.00 & 2.37 & 2.90 & 10.6 & $0.37:$ & 1.0 & $\cdots$ & 1.76 & $\cdots$ & 1.50 & 19.5 & 8.09 & 88.4 \\
\hline 9994 & Fe II? & & wk & $\ldots$ & wk & 1.96 & 5.25 & & 1.0 & $\cdots$ & 0.53 & $\cdots$ & & 1.56 & 2.28 & \\
\hline 10049 & P7 & 0.95 & 2.80 & abs & 1.20 & 3.62 & 20.5 & 0.66 & 0.46 & $\cdots$ & 1.47 & $\cdots$ & 0.33 & 5.57 & 9.25 & 7.38 \\
\hline
\end{tabular}

NOTE.-The term "sat." means the line or continuum in this region saturated the system. A colon means the uncertainty is greater than $30 \%$. The notation "wk" means the line is definitely present but oo weak to allow better than a $2 \sigma$ measurement. Ellipsis dots (..) mean that the line is not definitely present. The notation "abs " means the line is in absorption.

2 Units are $10^{-21} \mathrm{~W} \mathrm{~cm}^{-2}$ Typical $1 \sigma$ uncertainties are $+15 \%$ (absolute) and $+10 \%$ (relative). Paschen lines of $\mathrm{H}$ I are denoted by $\mathrm{P}$

3 We adopt the nomenclature of Pipher $e$ t al. 1976 instead of the redefinition of Gehrz et al. 1982

${ }^{4} \mathrm{P} 9$ is often blended with $\mathrm{Mg}$ II and other lines; see text. 
TABLE 2

EMISSION LINE FLUXES ${ }^{a}$

\begin{tabular}{|c|c|c|c|c|c|}
\hline $\begin{array}{l}\text { Line } \\
\text { (1) }\end{array}$ & $\begin{array}{l}\text { ID } \\
(2)\end{array}$ & $\begin{array}{c}\text { MWC } 297 \\
\text { (3) }\end{array}$ & $\begin{array}{c}\text { MWC } 349 \\
\text { (4) }\end{array}$ & $\underset{(5)}{\mathrm{LkH} \alpha} 101$ & $\begin{array}{l}\text { Notes } \\
(6)\end{array}$ \\
\hline $\begin{array}{l}6244 \ldots \ldots \ldots \ldots \\
6300 \ldots \ldots \ldots \ldots \\
6309 \ldots \ldots \ldots \ldots \\
6363 \ldots \ldots \ldots \ldots \\
6563 \ldots \ldots \ldots \ldots\end{array}$ & $\begin{array}{l}{\left[\begin{array}{ll}\mathrm{O} & \mathrm{I}\end{array}\right]} \\
{\left[\begin{array}{ll}\mathrm{O} & \mathrm{I}\end{array}\right]} \\
\mathrm{H} \alpha\end{array}$ & $\begin{array}{l}7.64(-20) \\
\ldots \\
3.06(-20) \\
6.51(-18)\end{array}$ & $\begin{array}{c}3.78(-20) \\
\ldots \\
5.39(-20) \\
\ldots \\
5.98(-18)\end{array}$ & $\begin{array}{l}\cdots \\
\cdots \\
\cdots \\
\cdots \\
\text { sat. }\end{array}$ & Could be $[\mathrm{S}$ III] \\
\hline $\begin{array}{l}6678 \ldots \ldots \ldots \ldots \\
7002 \ldots \ldots \ldots \ldots \\
7065 \ldots \ldots \ldots \ldots \\
7155+7172 \ldots \\
7281 \ldots \ldots \ldots \\
\ldots\end{array}$ & $\begin{array}{l}\mathrm{He}_{\mathrm{I}} \\
\mathrm{O}_{\mathrm{I}} \\
\mathrm{He}_{\mathrm{I}} \\
{\left[\mathrm{Fe}_{\mathrm{II}}\right]} \\
\mathrm{He}_{\mathrm{I}}\end{array}$ & $\begin{array}{c}<1.0(-20) \\
\ldots \\
\ldots \\
\ldots\end{array}$ & $\begin{array}{r}7.57(-20) \\
<9.0(-21) \\
2.61(-19) \\
1.35(-19) \\
7.09(-20)\end{array}$ & $\begin{array}{c}<1.0(-21) \\
2.00(-21) \\
1.94(-21) \\
\ldots\end{array}$ & Also [Ni I] \\
\hline $\begin{array}{l}7291 \ldots \ldots \ldots \ldots \\
7319+7330 \ldots \\
7378 \ldots \ldots \ldots \ldots \\
7452 \ldots \ldots \ldots \ldots \\
7516 \ldots \ldots \ldots \ldots\end{array}$ & $\begin{array}{l}{[\mathrm{Ca} \mathrm{II}]} \\
{\left[\begin{array}{ll}\mathrm{O} \\
\mathrm{II}\end{array}\right]} \\
{[\mathrm{Ni}} \\
{\left[\begin{array}{ll}\mathrm{Fe} & \mathrm{II}\end{array}\right]} \\
\mathrm{Fe}\end{array}$ & $\begin{array}{c}<2.3(-20) \\
\ldots \\
\ldots \\
\ldots \\
\ldots\end{array}$ & $\begin{array}{l}6.83(-20) \\
2.47(-19) \\
4.08(-20) \\
5.38(-20) \\
5.40(-20)\end{array}$ & $\begin{array}{c}<1.2(-21) \\
2.09(-21) \\
\ldots \\
\ldots \\
7.25(-21)\end{array}$ & \\
\hline $\begin{array}{l}7657 \ldots \ldots \ldots \ldots \\
7711 \ldots \ldots \ldots \ldots \\
7774 \ldots \ldots \ldots \ldots \\
7866 \ldots \ldots \ldots \ldots \\
7893 \ldots \ldots \ldots \ldots\end{array}$ & $\begin{array}{l}\mathrm{Fe} \text { II } \\
\mathrm{O}_{\mathrm{I}} \\
\mathrm{Fe}_{\mathrm{II}} ? \\
\text { (blend) }\end{array}$ & $\begin{array}{c}\ldots \\
\ldots \\
1.73(-19) \\
\ldots \\
\ldots\end{array}$ & $\begin{array}{l}5.41(-20) \\
4.71(-20) \\
2.48(-20) \\
9.18(-20) \\
1.13(-19)\end{array}$ & $\begin{array}{l}2.66(-21) \\
8.03(-21) \\
4.24(-21) \\
8.70(-21) \\
\ldots\end{array}$ & \\
\hline $\begin{array}{l}7917 \ldots \ldots \ldots \ldots \\
7975 \ldots \ldots \ldots \ldots \\
8000 \ldots \ldots \ldots \ldots \\
8100 \ldots \ldots \ldots \ldots \\
8230 \ldots \ldots \ldots \ldots\end{array}$ & $\begin{array}{l}{[\mathrm{Ti} \mathrm{II}]} \\
{[\mathrm{Ti} \mathrm{II}]} \\
\text { (blend) } \\
\text { (blend) } \\
\text { (blend) }\end{array}$ & $\begin{array}{l}\cdots \\
\cdots \\
\cdots \\
\cdots \\
\cdots\end{array}$ & $\begin{array}{c}\cdots \\
\ldots \\
4.65(-20) \\
9.79(-20) \\
1.93(-19)\end{array}$ & $\begin{array}{c}4.78(-21) \\
5.60(-21) \\
\ldots \\
1.36(-20) \\
1.20(-20)\end{array}$ & $\begin{array}{l}\text { See Herbig } 1971 \\
\text { [Ti II], [Cr II] } \\
\text { See Herbig } 1971\end{array}$ \\
\hline $\begin{array}{l}8287 \ldots \ldots \ldots \ldots \\
8358 \ldots \ldots \ldots \ldots \\
8446 \ldots \ldots \ldots \ldots \\
8498 \ldots \ldots \ldots \ldots \\
8542 \ldots \ldots \ldots \ldots\end{array}$ & $\begin{array}{l}\mathrm{Fe} \text { II? } \\
\mathrm{Fe} \text { II } \\
\mathrm{O}_{\mathrm{I}}+\mathrm{P} 17,18 \\
\mathrm{Ca}_{\mathrm{II}}+\mathrm{P} 16 \\
\mathrm{Ca}_{\mathrm{II}}+\mathrm{P} 15\end{array}$ & $\begin{array}{c}\ldots \\
2.47(-18) \\
2.05(-19) \\
2.31(-19)\end{array}$ & $\begin{array}{l}7.17(-20) \\
6.43(-20) \\
1.04(-18) \\
4.40(-19) \\
4.54(-19)\end{array}$ & $\begin{array}{l}7.91(-21) \\
\ldots \\
\text { sat. } \\
8.0(-21): \\
1.22(-20)\end{array}$ & $\begin{array}{l}\text { Paschen lines blended } \\
\text { U8358; Herbig } 1971 \\
\text { No Ca II in LkH } \alpha 101\end{array}$ \\
\hline $\begin{array}{l}8598 \ldots \ldots \ldots \ldots \\
8662 \ldots \ldots \ldots \ldots \\
8750 \ldots \ldots \ldots \ldots \\
8863 \ldots \ldots \ldots \ldots \\
8924 \ldots \ldots \ldots \ldots\end{array}$ & $\begin{array}{l}\mathrm{P} 14 \\
\mathrm{Ca} \text { II }+\mathrm{P} 13 \\
\mathrm{P} 12 \\
\mathrm{P} 11\end{array}$ & $\begin{array}{l}2.84(-19) \\
5.39(-19) \\
4.22(-19) \\
4.62(-19) \\
\ldots\end{array}$ & $\begin{array}{l}2.05(-19) \\
6.05(-19) \\
3.50(-19) \\
4.62(-19) \\
2.40(-19)\end{array}$ & $\begin{array}{l}1.85(-20) \\
3.20(-20) \\
3.38(-20) \\
4.60(-20) \\
1.74(-20)\end{array}$ & \\
\hline $\begin{array}{l}9014 \ldots \ldots \ldots \ldots \\
9069 \ldots \ldots \ldots \ldots \\
9125 \ldots \ldots \ldots \ldots \\
9229 \ldots \ldots \ldots \ldots \\
9391 \ldots \ldots \ldots \ldots \ldots\end{array}$ & $\begin{array}{l}\mathrm{P} 10 \\
{[\mathrm{~S} \text { III }]} \\
\text { P9+ blend }\end{array}$ & $\begin{array}{c}5.42(-19) \\
\ldots \\
\ldots \\
1.23(-18) \\
\ldots\end{array}$ & $\begin{array}{l}7.05(-19) \\
9.37(-19) \\
9.4(-20) \\
2.02(-18) \\
2.98(-19)\end{array}$ & $\begin{array}{l}6.23(-20) \\
1.58(-20) \\
1.83(-20) \\
1.91(-19) \\
1.93(-20)\end{array}$ & \\
\hline $\begin{array}{l}9460 \ldots \ldots \ldots \\
9532+9546 \ldots \\
9706 \ldots \ldots \ldots \ldots \\
9820 \ldots \ldots \ldots \ldots \\
9910 \ldots \ldots \ldots \ldots\end{array}$ & {$[\mathrm{S} \mathrm{III}]+\mathrm{P} 8$} & $\begin{array}{c}1.32(-18) \\
\ldots \\
\ldots \\
\ldots\end{array}$ & $\begin{array}{l}1.11(-19) \\
4.24(-18) \\
1.30(-19) \\
1.06(-19) \\
9.97(-20)\end{array}$ & $\begin{array}{c}2.67(-19) \\
\ldots \\
1.87(-20) \\
2.40(-20)\end{array}$ & \\
\hline $\begin{array}{l}9994 \ldots \ldots \ldots \ldots \\
10049 \ldots \ldots \ldots \ldots \\
10120 \ldots \ldots \ldots \\
10177 \ldots \ldots \ldots \\
10452 \ldots \ldots \ldots \\
\ldots\end{array}$ & $\begin{array}{l}\mathrm{Fe} \text { II? } \\
\mathrm{P} \\
\mathrm{He}\end{array}$ & $\begin{array}{l}\text { wk } \\
1.67(-18) \\
1.68(-19) \\
\ldots \\
\ldots\end{array}$ & $\begin{array}{c}8.38(-19) \\
2.78(-18) \\
\ldots \\
2.40(-19) \\
\ldots\end{array}$ & $\begin{array}{c}1.61(-19) \\
2.66(-19) \\
\ldots \\
4.67(-20) \\
1.56(-20)\end{array}$ & \\
\hline 10493 & & $\ldots$ & $1.16(-18)$ & $3.66(-19)$ & \\
\hline
\end{tabular}

a Fluxes are in units of $\mathrm{W} \mathrm{cm}^{-2}$ with powers of 10 in parentheses. Upper limits are $3 \sigma$. Other notes as for Table 1.

Notes for identifications. -Blank = Uncertainty in line wavelength precludes identification. Fe $\mathrm{II} ?=$ Coincidence with Fe II lines in Eta Car (Johansson 1977); secure identification awaits higher spectral resolution. 


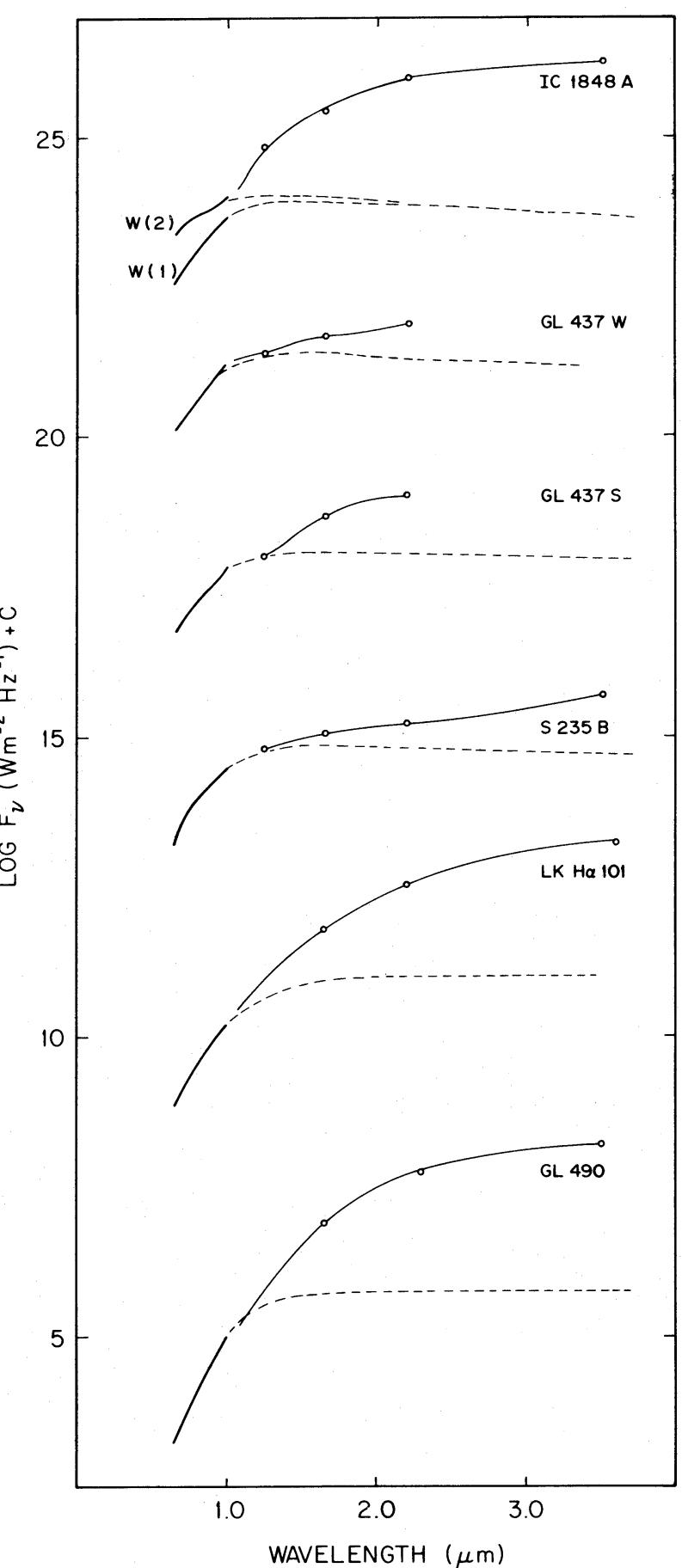

FiG. $2 a$

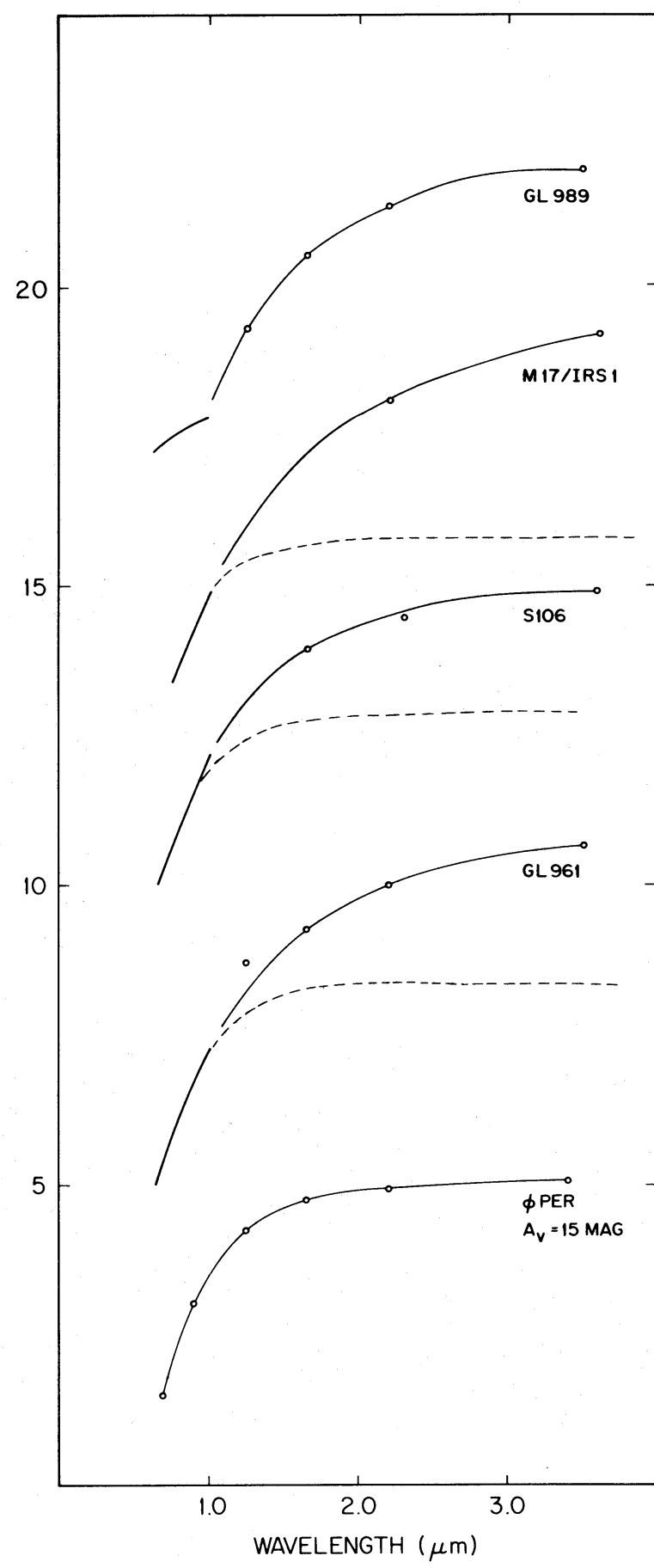

Fig. $2 b$

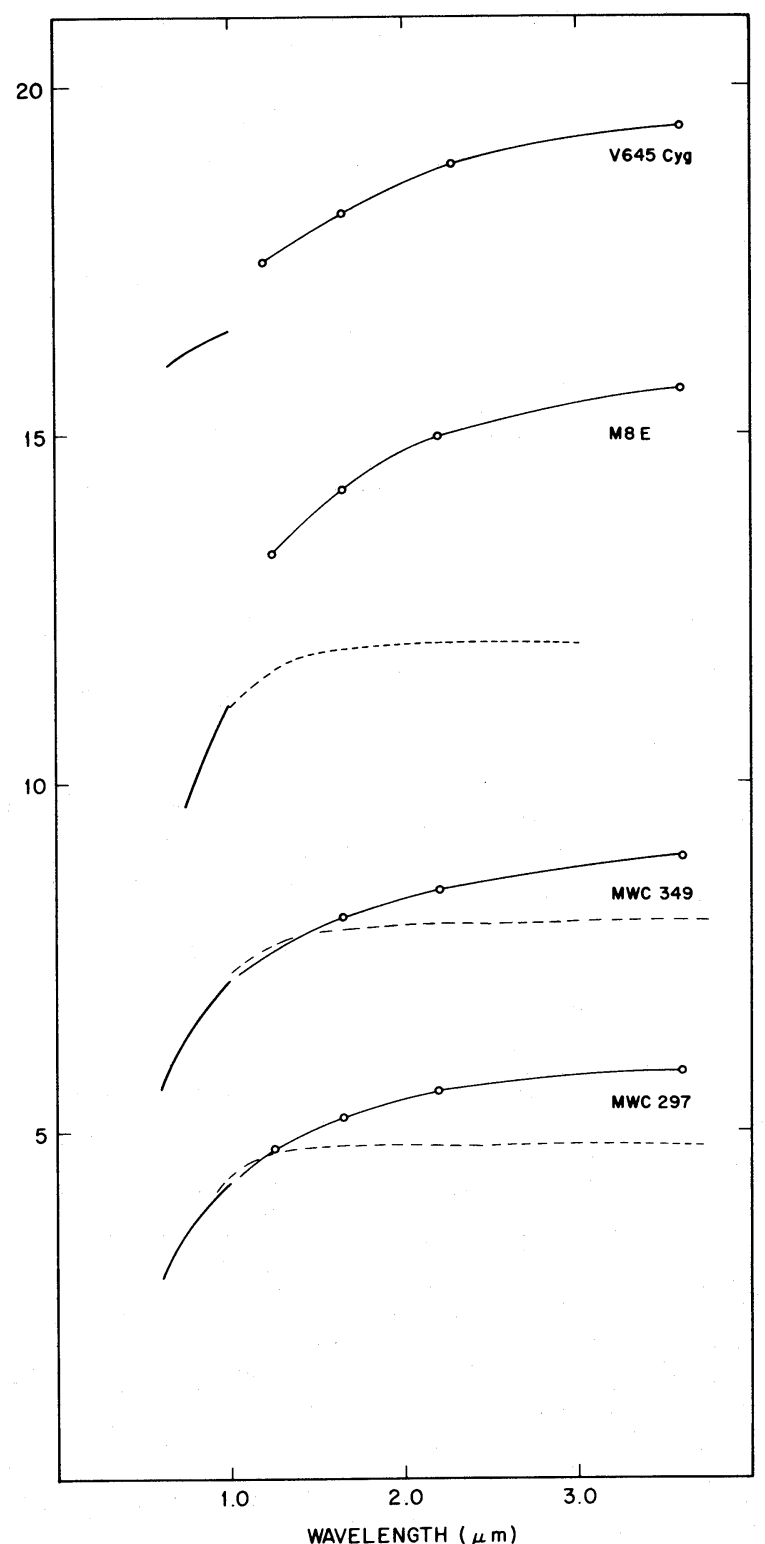

FIG. $2 c$

FIG. 2-Continuum energy distributions for the infrared objects, llustrating the generally good agreement between published broadband photometry (open circles; references in Table 7) and the present data. The flux density scale has an arbitrary zero-point; the actual continuum flux densities are given in Table 3. The dashed curves represent continuations of the model flux distributions used to fit the data between 0.6 and $1.0 \mu \mathrm{m}$. The $\phi$ Per curve shows how this Be star would look if it suffered 15 mag of visual extinction. 
TABLE 3

Continuum Flux Densities ${ }^{a}$

\begin{tabular}{|c|c|c|c|c|c|c|c|c|}
\hline \multirow[b]{2}{*}{ SOURCE } & \multicolumn{8}{|c|}{ WAVELENGTH $(\AA)$} \\
\hline & 6500 & 7000 & 7500 & 8000 & 8500 & 9000 & 9500 & 10000 \\
\hline IC $1848 \mathrm{~A}$ W(1) $\ldots \ldots \ldots \ldots$ & 17.40 & 16.88 & 16.37 & 16.00 & 15.80 & 15.33 & 15.08 & 14.70 \\
\hline IC $1848 \mathrm{~A} \mathrm{~W}(2) 2)$ & 15.55 & 15.14 & 14.83 & 14.59 & 14.46 & 14.31 & 14.09 & 13.90 \\
\hline GL $437 \mathrm{~W} \ldots \ldots \ldots$ & 16.18 & 15.68 & 15.22 & 14.80 & 14.44 & 14.00 & 13.64 & 13.35 \\
\hline GL $437 \mathrm{~S} .$. & 17.13 & 16.64 & 16.18 & 15.74 & 15.54 & 15.22 & 14.81 & 14.34 \\
\hline GL $490 \ldots \ldots \ldots \ldots \ldots \ldots$ & 18.29 & 17.45 & 16.68 & 16.02 & 15.54 & 15.00 & 14.47 & 13.83 \\
\hline $\mathrm{LkH} \propto 101 \ldots$ & 14.38 & 13.78 & 13.13 & 12.53 & 12.20 & 11.69 & 11.25 & 10.91 \\
\hline S235B $\ldots \ldots \ldots \ldots \ldots$ & 15.65 & 15.00 & 14.43 & 13.95 & 13.72 & 13.31 & 13.06 & 12.71 \\
\hline GL $961 \ldots \ldots \ldots \ldots$ & 21.4 & 20.3 & 19.34 & 18.42 & 17.66 & 16.90 & 16.29 & 15.68 \\
\hline GL 961 FAN & 16.72 & 16.28 & 15.91 & 15.60 & 15.28 & 15.05 & 14.76 & 14.53 \\
\hline GL 989 (1) $\ldots \ldots \ldots \ldots \ldots$ & 16.95 & 16.69 & 16.43 & 16.21 & 16.00 & 15.90 & 15.80 & 15.66 \\
\hline GL 989 (2) & 16.09 & 15.75 & 15.41 & 15.13 & 14.91 & 14.78 & 14.53 & 14.29 \\
\hline M8E .............. & & & 19.7 & 18.9 & 18.2 & 17.4 & 16.8 & 16.1 \\
\hline M17/IRS $1 \ldots \ldots \ldots \ldots$ & 21.9 & 21.0 & 20.3 & 19.57 & 18.86 & 18.28 & 17.65 & 16.77 \\
\hline MWC $297 \ldots \ldots \ldots \ldots \ldots$ & 11.06 & 10.48 & 9.91 & 9.44 & 9.19 & 8.99 & 8.75 & 8.27 \\
\hline S106/IRS $3 \ldots \ldots \ldots \ldots \ldots$ & 18.84 & 17.88 & 17.09 & 16.16 & 15.57 & 14.81 & 14.18 & 13.41 \\
\hline V645 Cyg... & 13.94 & 13.73 & 13.49 & 13.30 & 13.17 & 13.05 & 12.93 & 12.74 \\
\hline MWC $349 \ldots \ldots \ldots \ldots \ldots$ & 11.75 & 11.16 & 10.60 & 9.98 & 9.68 & 9.37 & 9.06 & 8.50 \\
\hline NGC 7538/IRS $2 \ldots \ldots \ldots$ & 20.6 & 18.78 & 17.73 & 16.75 & 16.10 & 15.33 & 14.78 & 14.04 \\
\hline
\end{tabular}

strong [S III] lines at $\lambda \lambda 9069,9532$. These lines are also present in the spectrum of M17/IRS 1 . The $\lambda 9532$ line in NGC 7538/ IRS 2 is about 12 times stronger than P7 ( $\lambda 10049)$ uncorrected for reddening, and roughly 25 times stronger if corrected for $A_{V}{ }^{\prime}=14.5 \mathrm{mag}$ (Table 4). This ratio is not very different from that found in the Orion Nebula (H. M. Johnson 1968), which is consistent with NGC 7538/IRS 2 being a compact $\mathrm{H}$ II region excited by an early-type star. Information on other ionization stages of sulfur is lacking, and since $\mathrm{S}^{++}$is abundant for a rather wide range of $T_{\text {eff }}$ (see, e.g., Rank et al. 1978), it is not possible to restrict the temperature of the exciting star. Nevertheless, the great strength of the [S III] lines suggests that $T_{\text {eff }}$ is closer to $30,000 \mathrm{~K}$ than to $40,000 \mathrm{~K}$. This estimate is not consistent with the bolometric luminosity of $2.5 \times 10^{5} L_{\odot}$ if the star is on the zero-age main sequence (Panagia 1973). Measurements of the infrared fine-structure lines of $[\mathrm{S} \mathrm{IV}]$ are required to study this problem further.

There are no other very strong lines, either in emission or absorption, that characterize the spectra of the embedded sources in the $0.6-1.0 \mu \mathrm{m}$ range. There are, however, two additional features present in several of the spectra. The H I P9 line at $\lambda 9229$ is clearly too strong in many of the objects to be compatible with other Paschen lines in its vicinity (see Fig. 1), and also appears broader than the instrumental resolution. Johnson, Wisniewski, and Fäy's (1978) spectra of four O-B stars clearly show a similar feature in $\phi$ Per (B2pe), P Cygni (B1ep), and $\gamma$ Cas (B0 IVe). The widths of this feature in these Be stars $(\sim 100 \AA$ FWHM $)$ are consistent with those in Figure 1. Johnson et al. identified the feature as due to a blend of P9 with lines of $\mathrm{Mg}$ II $(\lambda \lambda 9217,9243), \mathrm{O}_{\mathrm{I}}(\lambda 9266), \mathrm{Fe}$ II $(\lambda 9214), \mathrm{He}$ I ( $\lambda 9210)$, and $\mathrm{Ne}$ I ( $\lambda 9202)$. This characteristic feature is not known to occur in any other stars, but the data in this spectral range are limited.

The second feature is a line near $\lambda 9994$ which is moderately strong in the spectra of LkH $\alpha 101$, GL 490, GL 961 FAN, and
MWC 349, and is also detectable in S106/IRS 3, S235B, GL 989 (2), V645 Cyg, and GL 437S. This line also appears in $\phi$ Per, where it rivals P7 in strength, and weakly in P Cyg and $\gamma$ Cas, but not in the spectra of ordinary O-B stars. It is also present in several other peculiar emission-line objects with infrared excesses (Andrillat and Swings 1976). The line is probably the same as that seen in $\eta$ Car and identified by Johansson (1977) as due to Fe II.

Finally, MWC 349 and $\mathrm{LkH} \alpha 101$ display a number of weak emission lines in this spectral region, many of which are also apparent in the $\lambda<8600 \AA$ spectrum of $\mathrm{LkH} \alpha 101$ due to Herbig (1971). At the low resolution of our spectra we are unable to improve upon this situation and reserve discussion of these features to a later paper where we present higher resolution spectra of these objects. The equivalent widths of the stronger and less blended lines in $\mathrm{LkH} \alpha 101$ agree well with those of Herbig (1971).

From the point of view of spectral classification, the nearinfrared spectra presented in Figure 1 share many features in common with Be stars. For the objects that are not compact $\mathrm{H}$ II regions, there are in fact no glaring differences between the spectra of the embedded sources and those of Be stars. We wish to stress, however, that these parallels should not be interpreted to mean that the embedded sources simply are Be stars; such a conclusion would imply an evolutionary phase for the embedded objects that is far more advanced than could be reconciled with many other indications of their youth. Rather, it seems clear that the embedded objects possess circumstellar envelopes that have similar physical conditions to those in other objects that have been studied in more detail.

\section{b) The Continua}

The $0.6-1.0 \mu \mathrm{m}$ continua are red and free of strong absorption features. The Paschen jump is strongly in emission in S235B and MWC 297, and in fact is present or weakly present 
TABLE 4

SOURCE EXTINCTIONS AND LUMINOSITIES

\begin{tabular}{|c|c|c|c|c|c|}
\hline & & & $\mathrm{AB}(1$ & $\mu \mathrm{m})$ & \\
\hline $\begin{array}{l}\text { Source } \\
\text { (1) }\end{array}$ & $\begin{array}{l}{ }^{A} v^{\prime} \\
(2)\end{array}$ & $\begin{array}{l}\mathrm{L} / \mathrm{L}_{\odot} \\
(3)\end{array}$ & $\begin{array}{l}\text { predicted } \\
\text { star alone } \\
\text { (4) }\end{array}$ & $\begin{array}{c}\text { measured } \\
\text { (5) }\end{array}$ & $\begin{array}{c}\text { Notes } \\
(6)\end{array}$ \\
\hline $\begin{array}{l}\text { IC } 1848 \mathrm{~A} \text { W(1) } \\
\text { IC } 1848 \mathrm{~A} \text { W(2) }\end{array}$ & $\begin{array}{l}7.7 \\
5.2\end{array}$ & $2.2 \times 10^{4}$ & 12.4 & $\begin{array}{l}14.7 \\
13.9\end{array}$ & $1 \mu \mathrm{m}$ data do not include point source. \\
\hline $\begin{array}{l}\text { GL } 437 \mathrm{~W} \\
\text { GL } 437 \mathrm{~S}\end{array}$ & $\begin{array}{l}7.7 \\
7.5\end{array}$ & $3.0 \times 10^{4}$ & 12.7 & $\begin{array}{l}13.4 \\
14.3\end{array}$ & Multiple IR source. \\
\hline GL 490 & 11.5 & $1.4 \times 10^{3}$ & 13.5 & 13.8 & OK \\
\hline Lk $\mathrm{H} \alpha \quad 101$ & 9.7 & $1.2 \times 10^{4}$ & 11.2 & 10.9 & OK \\
\hline S235B & 8.4 & $1.0 \times 10^{3}$ & 14.1 & 12.7 & Source observed brighter than expected at $1 \mu \mathrm{m}$. \\
\hline GL 961 & 15.3 & $5.5 \times 10^{3}$ & 14.8 & 15.7 & OK \\
\hline M8E & (15) & $2.5 \times 10^{4}$ & 14.0 & 16.1 & See text \\
\hline M17/IRS 1 & 14.5 & $3 \times 10^{3}$ & 16.2 & 16.8 & OK \\
\hline MWC 297 & 7.9 & $\cdots$ & $\cdots$ & 8.3 & $\cdots$ \\
\hline S106/IRS3 & 13.5 & $2 \times 10^{4}$ & 11.6 & 13.4 & See text \\
\hline V645 Cyg & 4.2 & $9 \times 10^{4}$ & 12.4 & 12.7 & OK \\
\hline MWC 349 & 8.8 & $1.5 \times 10^{4}$ & 12.7 & 8.5 & Strong shell? See text \\
\hline NGC $7538 /$ IRS 2 & 14.5 & $2.5 \times 10^{5}$ & 14.1 & 14.0 & OK \\
\hline
\end{tabular}

NoTE.-The extinction for M8E is uncertain.

in all the spectra except GL $437 \mathrm{~W}$ and NGC 7538/IRS 2, two compact $\mathrm{H}$ II regions. The Paschen jump is not seen in emission in the spectra of normal early-type stars. However, it is seen in emission in some Be stars (e.g., Scargle et al. 1978) and the Herbig Ae-Be stars (Garrison 1978), where some fraction of the continuum emission arises in a circumstellar shell. Defining a parameter to measure the size of the Paschen jump as $\Delta p=$ $-2.5 \log \left(F_{8204+} / F_{8204-}\right)$, where the $F$ 's are the flux densities on either side of the jump, we find $\Delta p \approx 0.2 \mathrm{mag}$ for S235B and also $0.2 \mathrm{mag}$ for the Be star $\phi$ Per which has the largest $\Delta p$ of the Be stars measured by Johnson, Wisniewski, and Fäy (1978). As discussed below, unambiguous interpretation of the Paschen jump parameter is not possible without knowledge of the underlying photospheric energy distribution and other information, but it is apparent that the Paschen continuum emission in the embedded sources is comparable to, or weaker than, that seen in at least one Be star, and is consistent with a circumstellar shell origin.

The Paschen jump is also seen in emission in the recombination spectrum of optically thin ionized gas. The $\mathrm{H}$ I line equivalent widths in the embedded source spectra are far too small to arise solely from optically thin hydrogenic free-free plus bound-free emission, and an additional continuum source must be present. As any hot dust contribution is likely to be negligible at these wavelengths, it is more likely that we are seeing emission from a circumstellar shell and/or the photosphere of the early-type star that is inferred to be present to supply the total luminosity measured in the far-infrared. In general, the shell may have contributions from optically thick and optically thin gas emission at unknown temperatures. If the shell emission is optically thin, the small sizes of the Paschen jumps indicate that in all cases the observed spectra are dominated by stellar photospheric light, varying approximately as $v^{2}$. We note that in $\phi$ Per photospheric light also dominates over the shell emission in this spectral region (Gehrz, Hackwell, and Jones 1974). If the shell is optically thick, it emits as a blackbody, and if its temperature exceeds $\sim 10^{4} \mathrm{~K}$, the spectral energy distribution will also vary approximately as $v^{2}$. It is also possible that for objects deeply embedded in dust the continuum is affected by scattering; this is clearly the cases for IC 1848A and GL 989, and to a lesser extent S106/IRS 3.

As a first approximation to fitting the energy distribution and thereby deriving the amount of reddening, we have ignored scattering and have modelled the observed continua as the sum of a hydrogenic free-free plus bound-free shell component $\phi_{v}\left(\tau_{v}^{s}\right)$ having optical depth $\tau_{v}^{s}$, plus continuous emission $F_{v}^{*}$ from the photosphere of a hot star. Following Scargle et al. (1978), the combined (unreddened) flux density $F_{v}$ can be expressed as

$$
F_{v}=\left(1-f^{s}\right) F_{v}^{*} \exp \left(-\tau_{v}^{*}\right)+f^{s} \phi_{v}\left(\tau_{v}^{s}\right),
$$

where $f^{s}$ is the fractional contribution of shell emission at a fiducial wavelength and the stellar flux is attenuated by optical depth $\tau_{v}^{*}$ through the shell. Explicit expressions for $\phi_{v}(\tau)$ are given by Scargle et al. (1978) for isothermal slabs and isother- 
mal sphere models. Our results are not affected by the choice of geometry. We have simply assumed that the shell temperature is $10^{4} \mathrm{~K}$ and that the stellar energy distribution is that of $25,000 \mathrm{~K}$ blackbody; in the Rayleigh-Jeans limit the continuum slope is independent of temperature. The absorption optical depth in the shell to the stellar emission may be quite small $\left(\tau_{v}^{*}<0.1\right)$ if the shell is a thin disk; we have further simplified the model by neglecting attenuation of the starlight by the shell. The reddening law used was the van de Hulst curve 15 (H. L. Johnson 1968), which is close to the Whitford (1958) curve. For each object, the continuum flux density at $8500 \AA$ was forced to match the observed value while the relative contributions of stellar and circumstellar emission, the optical depth at $8500 \AA$ in the shell, and the extinction to the source were varied to obtain the best fit, as judged by eye, to the observed continuum. Since for high optical depths both contributors have approximately $v^{2}$ energy distributions, the star and shell cannot be uniquely separated. However, this fact does allow a reasonably good estimate of the extinction to be made, if scattering does not strongly distort the spectral energy distribution. Table 4 gives the results, where the visual extinctions in magnitudes, $A_{V}{ }^{\prime}$, are listed. The simple assumed form of the continuum was found to fit the data well for every object, with typical deviations being less than $15 \%$ throughout each spectrum. Reasonable fits to the Paschen jump emission were obtained by suitably choosing the shell optical depth for an assumed stellar contribution. The exact details of the fit in the region of the Paschen jump had minimal effect on the derived extinctions. Nevertheless, it is difficult to gauge the uncertainties in the tabulated extinctions because of the uncertain shell temperatures and role of scattering.

The probable importance of a photospheric contribution to the observed continuum raises the possibility of detecting photospheric absorption lines. However no strong lines, except for hydrogen lines, occur in early-type stars in this spectral region (Johnson 1978), so no information of this kind is obtainable from the spectra.

If the photosphere of the embedded star dominates the $1 \mu \mathrm{m}$ continuum, we would expect rough agreement between the observed $1 \mu \mathrm{m}$ flux densities and those inferred from the bolometric luminosities and the $1 \mu \mathrm{m}$ extinction. To transform the measured bolometric luminosities (from far-infrared photometry) to $1 \mu \mathrm{m}$ flux densities we used the $M_{1 \mu \mathrm{m}}-M_{V}$ colors appropriate to the Kurucz (1979) $25,000 \mathrm{~K}$ model atmosphere together with Panagia's (1973) tables of $M_{V}$ and $\log L / L_{\odot}$ for early type stars, under the assumption that the embedded sources lie on the ZAMS. The source distances were taken from Wynn-Williams (1982) for all sources except S106 for which a distance of 600 pc was assumed (Staude et al. 1982). Table 4 contains the observed and predicted $1 \mu \mathrm{m}$ continuum magnitudes. Given the assumptions that enter this comparison (reddening law, the different spatial scales of the far-infrared and $1 \mu \mathrm{m}$ data), agreement to within a factor of 3 should be considered reasonable, and the notes in Table 4 indicate that this is the usual case. The agreement for NGC 7538/IRS 2 seems fortuitous in view of the multiple nature of the source and the large beam of the far-infrared data. One cannot expect good agreement for GL 437 because this source is also complex. For IC 1848A and M8E, the poor agreement reflects the fact that we are not seeing the whole $1 \mu \mathrm{m}$ continuum (see Appendix).

The observed $1 \mu \mathrm{m}$ continuum for MWC 349 is much larger than expected on the basis of the total luminosity. Distance uncertainties affect both the measured and predicted luminosities and cannot be responsible. It is possible that only a small fraction of the primary stellar flux is converted to dust emission, but it seems more likely that shell emission dominates the continuum. A model that fits the data then consists of a shell $(T \sim 10,000 \mathrm{~K})$ with $\tau(8500 \AA)=1,3 \%$ of the continuum due to a hot star, and $A_{V}=8.5 \mathrm{mag}$. The shell optical depth must be large enough to produce a small Paschen jump, and, as expected, the extinction value is close to the Table 3 value of $8.8 \mathrm{mag}$.

The discrepancies for S106/IRS 3 and S235B are large, and in opposing senses. In S106, the observed total luminosity predicts a $1 \mu \mathrm{m}$ flux that is 5 times larger than observed. Other far-infrared sources in the vicinity can hardly account for a discrepancy of this magnitude if IRS 3 is the exciting star, as seems secure (Gehrz et al. 1982). A simple explanation is that our value of $A_{V}{ }^{\prime}$ is too low, perhaps because the underlying light is strongly scattered as it leaves the vicinity of the star. Indeed, IRS 3 appears fuzzy on the red photograph of Eiroa et al. (1979), and we have included a bluish wisp of nebulosity in our spectrum as discussed in the Appendix. Gehrz et al. (1982) have also suggested scattering close to IRS 3 in discussing the reddening value of Eiroa, Elsässer, and Lahulla (1979).

The observed $1 \mu \mathrm{m}$ flux density of S235B is $\sim 4$ times brighter than expected on the basis of its total luminosity. A possible explanation is that the dust distribution around the source allows the escape of most of the photons before they can be absorbed. If true, the line excitation problem (Thompson 1982; Thompson, Thronson, and Campbell 1983) is removed for this source; the $\log L / L_{\odot}$ value found from the $1 \mu \mathrm{m}$ flux places S235B squarely on the theoretical relationship of luminosity versus $N_{e}^{2} V$ for Panagia's models (see Thompson 1982, Fig. 1). However, other explanations are certainly possible. For example, another redder star could contribute to the observed $1 \mu \mathrm{m}$ continuum, or the shell emission could dominate, as in the case of MWC 349.

The continuations of the two-component models discussed above into the near infrared region are plotted as dashed lines in Figure 2. In all cases they fall below the observed nearinfrared flux distributions longward of about $1 \mu \mathrm{m}$. The Be star $\phi$ Per is representative of Be stars with strong infrared excesses (Mendoza 1982; Feinstein 1982), and its energy distribution, artificially reddened by an amount corresponding to $A_{V}=15$ mag, is also shown in Figure 2. Although this star has a large infrared excess $(K-L=0.52)$, it can be seen that the embedded sources have even steeper near-infrared continua than this; the excess emission in the $2 \mu \mathrm{m}$ region in the embedded sources is therefore likely to be due to hot dust which swamps the gaseous shell emission.

\section{c) The Hydrogen Lines}

It is a simple matter to show that Case $\mathrm{B}$ recombination predictions (Brocklehurst 1971; Giles 1977) do not produce satisfactory agreement with the Paschen line decrements and $\mathrm{Br} \alpha$ and $\mathrm{Br} \gamma$ line strengths in the embedded sources. A convenient way to illustrate this problem, which does not depend on knowing the source extinctions, is via the diagram of WynnWilliams (1983). Figure 3 shows the $5 \mathrm{GHz}$ radio flux (assumed optically thin), predicted on the basis of Case $B$ recombination theory, for each measured line in the Balmer, Paschen, Brackett, and Pfund series plotted against the relative extinction, $A_{\lambda} / A_{V}$, of the line in question. The open circles correspond to the P9 line (and in some cases also the P8 line), which 


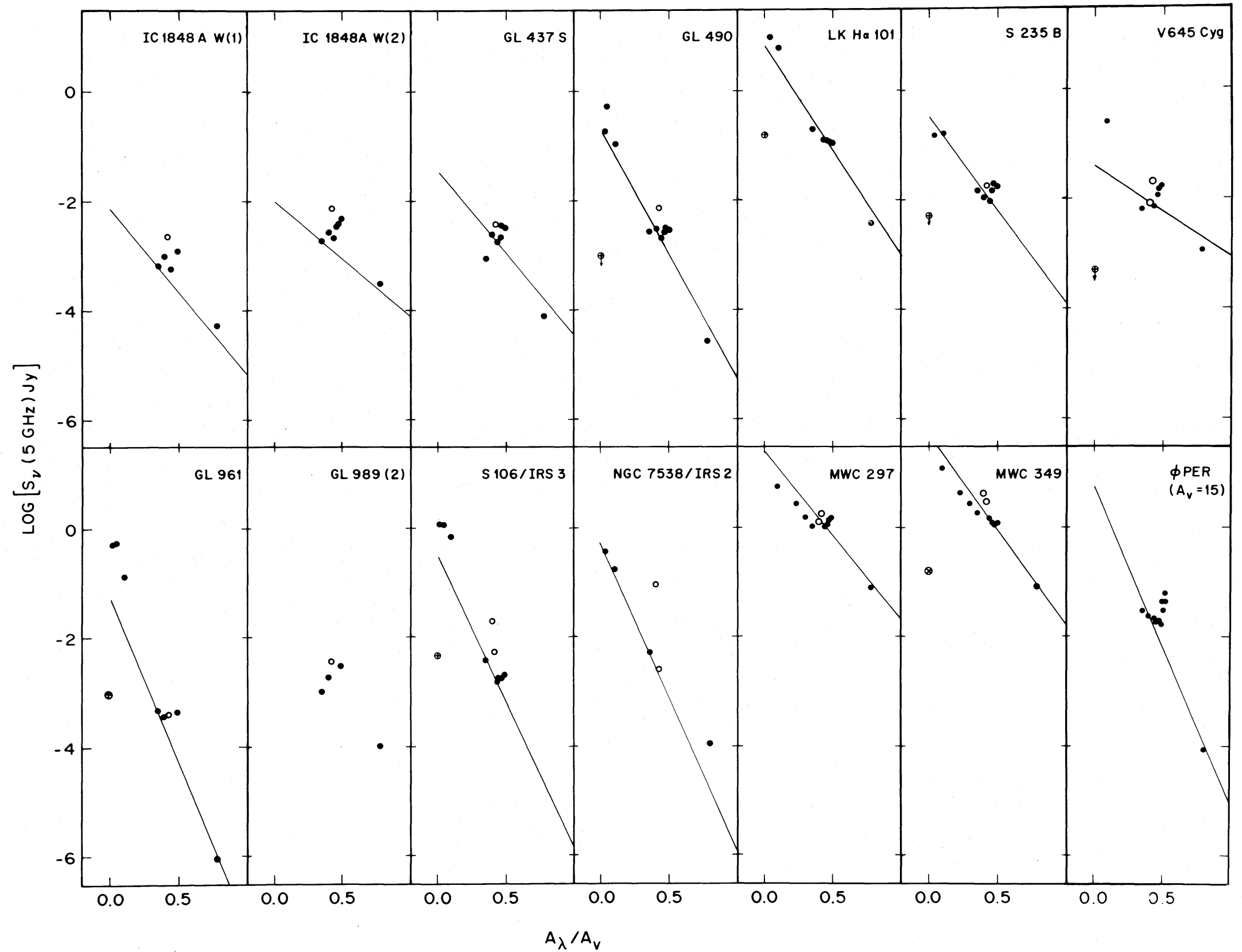

FIG. 3.-Each point represents a prediction for the optically thin $5 \mathrm{GHz}$ radio flux density based on the observed $\mathrm{H}$ i line flux if all the line fluxes follow Case $\mathrm{B}$ recombination theory. The abscissa is the normalized reddening law, so that were Case B to apply, all the points would lie on a straight line whose slope gave the amount of reddening. The open circles are the P8 and P9 lines which can be blended with other lines. Measured radio fluxes are the circled crosses at $A_{2} / A_{V}=0$. Error bars are comparable to the size of the points in this plot. 
is blended with other lines (see $\S$ III $a$ ), and is plotted only to emphasize this fact. For Case $\mathbf{B}$ recombination theory and a standard reddening law, all the points should fall on a straight line whose slope measures the extinction, $A_{V}$. The straight line drawn on each frame corresponds to the reddening found from the continuum slope above. Figure 3 shows that for no object, except the compact $\mathrm{H}$ II region NGC 7538/IRS 2 (see Appendix) and perhaps the visible star MWC 349, do the points fall cleanly on either the predicted line or any other straight line corresponding to a different reddening. The new Paschen line data, which are represented by the small cluster of points at $A_{\lambda} / A_{V} \sim 0.45$ in each plot, thus show that for the compact embedded sources the hydrogen lines are not formed in an optically thin Case B plasma. This strongly reinforces a similar conclusion based on the $\mathrm{Br} \alpha, \mathrm{Br} \gamma$, and $\mathrm{Pf} \gamma$ lines alone for GL 490 (Wynn-Williams 1983).

The similar character of each cluster of Paschen line data points should be noted: the higher Paschen lines are systematically strong compared to the lower lines. It is of interest to compare the Paschen line decrements with those seen in other emission-line objects. As an example, the $5 \mathrm{GHz}$ predictions from $\mathrm{H} \alpha$ and the Paschen series lines for the Be star $\phi$ Per are also plotted in Figure 3. The Paschen line strengths were taken from Johnson, Wisniewski, and Fäy's (1978) list of equivalent widths and converted to fluxes via the continuum energy distribution of Mendoza (1982). To further clarify the comparison, the Be star line strengths were artificially reddened by a further $A_{V}=15$ mag. It is clear that qualitatively the same effect is seen in the Be star $\phi$ Per as in the embedded objects. The Paschen lines deviate systematically from recombination theory to give a characteristic minimum in the distribution of points near P15. The higher Paschen lines are again systematically too strong compared to the lower lines. Qualitatively the same pattern of Paschen line points as in Figure 3 is obtained for a model in which all the measured Paschen lines have the same flux. This result is discussed in $§$ IV.

\section{d) The O I Lines}

At the present spectral resolution the hydrogen P17 and P18 lines are blended with $\mathrm{O}_{\mathrm{I}} \lambda 8446$, so to correct for these lines we have assumed that the dereddened line fluxes for P17 and P18 are the same as for P14, as with optically thick emission, rather than using the Case B recombination ratios which are surely inappropriate (Fig. 3). The reddening values adopted to correct the ratios of the $\lambda 8446$ line to other lines were those derived from our continuum fitting procedure (Table 4).

There are three possible mechanisms for exciting the $\lambda 8446$ line in gaseous nebulae, and each gives rise to a different set of permitted lines. These are pure recombination, continuum fluorescence (Grandi 1975), and Ly $\beta$ fluorescence (Bowen 1947; Grandi 1975, 1980; Strittmatter et al. 1977). In pure recombination the $\mathrm{O}_{\mathrm{I}} 27774$ line, which is the quintet analog of $\lambda 8446$, should be present in the spectra with an unreddened strength $\sim 1.7$ times that of the $\lambda 8446$ triplet line. The spectra have been carefully inspected at the position of the 27774 line and upper limits to the line strengths estimated. The values in Tables 1 and 2 show that in all cases the $\lambda 7774 / \lambda 8446$ ratios are less than 0.4 after correcting for the amount of reddening corresponding to the values in Table 4 . This safely eliminates pure recombination as the $\lambda 8446$ line formation mechanism in the embedded objects.

Distinguishing between $\operatorname{Ly} \beta$ fluorescence and continuum, or starlight, excitation from the present data is more difficult. In the $\operatorname{Ly} \beta$ fluorescence mechanism, upper $\mathrm{O}_{\mathrm{I}}$ levels are excited because of a coincidence in the wavelengths of the $\mathrm{H}_{\mathrm{I}} \mathrm{Ly} \beta$ $\lambda 1025.72$ and the $O$ I $\lambda 1025.76$ transitions. The subsequent cascade emits in the $\lambda 11287\left(3 p^{3} P-3 d^{3} D\right), \lambda 8446\left(3 s^{3} S-3 p{ }^{3} P\right)$, and $\lambda 1302\left(2 p^{3} P-3 s^{3} S\right)$ transitions. In direct starlight excitation additional $\mathrm{O}_{\mathrm{I}}$ ultraviolet transitions participate in the excitation and many more visible and infrared transitions emit. These processes have been discussed in detail by Grandi (1975) and Strittmatter et al. (1977). The emission lines of importance in establishing that starlight excitation is dominant occur at $\lambda \lambda 7002,7254$, and 13164 . The expected ratios of these lines to $\lambda 8446$ depend on the assumed form of the exciting stellar continuum. If the stellar spectrum has $\mathrm{Ly} \beta$ in absorption, the $\lambda \lambda 11287,8446$, and 1302 lines will be weaker. Assuming Ly $\beta$ absorption in the exciting star, Grandi (1975) predicted $\lambda 7002 /$ $\lambda 8446 \approx 0.4$ and $\lambda 7254 / \lambda 8446 \approx 0.1$ in the Orion Nebula. Inspection of our spectra at the positions of these lines gives upper limits and, in three cases, detections. Tables 1 and 2 lists the $3 \sigma$ upper limits and detections for $\lambda 7002$, the more sensitive discriminant. From the dereddened $\lambda 7002 / \lambda 8446$ line ratios we are able to eliminate starlight excitation as the dominant $\lambda 8446$ excitation mechanism in GL 490, GL 989(2), GL 437W, GL 437S, S235B, MWC 297, MWC 249, and probably S106/IRS 3 and GL 961 FAN. Good evidence for starlight excitation is found for IC $1848 \mathrm{~A} \mathrm{~W}(1)$ and $\mathrm{W}(2)$, where the $\lambda 7002 / \lambda 8446$ ratios are of order unity.

A test of the $\operatorname{Ly} \beta$ versus continuum fluorescence mechanisms that is more sensitive than the $\lambda 7002 / \lambda 8446$ ratio for heavily reddened objects is provided by the lines at $\lambda 11287$ and $\lambda 13164$ (neither of which lies within our spectral coverage). The $\lambda 11287$ line feeds the $\lambda 8446$ transition, and so its strength should be comparable to that of $\lambda 8446$ in the $\operatorname{Ly} \beta$ case. The $\lambda 13164$ line, on the other hand, can only be excited by continuum photons, not by $\operatorname{Ly} \beta$ photons. The $\lambda 11287$ line is seen in the visible young stellar objects MWC 349, MWC 297, and $\mathrm{LkH} \alpha 101$ (Thompson et al. 1977), whereas the $\lambda 13164$ line is absent in all three objects. If $\mathrm{O}_{\mathrm{I}} \lambda 8446$ formation in the embedded sources and $\mathrm{O}_{\mathrm{I}} \lambda 8446$ formation in these visible young stellar objects are analogous, then we have further evidence that the $\mathrm{O}_{\mathrm{I}}$ $\lambda 8446$ line in the embedded source spectra arises from $\mathrm{Ly} \beta$ fluorescence.

It is natural to discuss the strength of the $\lambda 8446$ line in terms of the $\lambda 8446 / \mathrm{H} \alpha$ ratio, because both lines are fed by conversion of $\operatorname{Ly} \beta$ photons. The dereddened values for $\lambda 8446 / \mathrm{H} \alpha$ lie in the range $0.01-0.06$, but are uncertain mostly because of reddening uncertainties. The sensitivity of $\lambda 8446 / \mathrm{H} \alpha$ to $A_{V}{ }^{\prime}$ is such that if $A_{V}^{\prime}$ is increased by $5 \mathrm{mag}$, the corrected value of $\lambda 8446 / \mathrm{H} \alpha$ is decreased by a factor of 3 .

\section{e) The Ca II Lines}

The $\mathrm{Ca}$ II triplet lines arise from the same upper level $\left(4^{2} \mathrm{P}\right)$ as the strong $\mathrm{H}$ and $\mathrm{K}$ resonance lines at 3969 and $3934 \AA$. The lower levels of the triplet transitions are metastable and decay to ground in forbidden transitions at a rate of $\sim 1.3 \mathrm{~s}^{-1}$. High optical depth in the Ca triplet is possible because of the metastability of the lower levels, and as they are pumped by absorption of $\mathrm{H}$ and $\mathrm{K}$ photons, the triplet lines can occur in emission even when $\mathrm{H}$ and $\mathrm{K}$ are in absorption. It is necessary to first correct the observed line strengths in Tables 1 and 2 for the blended Paschen 13, 15, and 16 lines of $\mathrm{H}$ I. The line strengths were first corrected for reddening using the $A_{V}{ }^{\prime}$ values in Table 4 , and then for the Paschen lines by subtracting the P14 line strength from each blend. Table 5 lists the ratios of the 
TABLE 5

Ca II Line Parameters

\begin{tabular}{|c|c|c|c|c|c|}
\hline $\begin{array}{l}\text { Source } \\
\text { (1) }\end{array}$ & $\begin{array}{c}I(\lambda 8542) \\
\left(\mathrm{W} \mathrm{cm}^{-2}\right) \\
(2)\end{array}$ & $\begin{array}{c}\mathrm{W}_{\lambda}(\lambda 8542) \\
\text { (A) } \\
\text { (3) }\end{array}$ & $\begin{array}{c}\log I(\lambda 8498) / \\
I(\lambda 8542) \\
(4)\end{array}$ & $\begin{array}{c}\log I(\lambda 8662) / \\
I(\lambda 8542) \\
(5)\end{array}$ & $\begin{array}{c}I(\lambda 8542) / \\
I(\lambda 7291) \\
(6)\end{array}$ \\
\hline IC $1848 \mathrm{~A}$ W(1) & $5.1(-20)$ & 20.3 & -0.12 & -0.04 & $>3$ \\
\hline IC $1848 \mathrm{~A}$ W(2) & $4.4(-20)$ & 17.0 & -0.24 & -0.04 & $>8.9$ \\
\hline GL 437S .............. & $1.0(-20)$ & 3.7 & -0.27 & 0.44 & $>2$ \\
\hline GL $490 \ldots \ldots \ldots \ldots \ldots$ & $8.9(-20)$ & 4.9 & 0.01 & 0.24 & $>3$ \\
\hline GL $961 \ldots \ldots \ldots \ldots \ldots$ & $7.7(-20)$ & 5.4 & -0.02 & 0.21 & $>0.2$ \\
\hline M8E & $2.2(-19)$ & 30. & $\sim 0$ & $\sim 0$ & $>0.2$ \\
\hline GL 989 (1) .... & $8.2(-22)$ & 16.2 & -0.04 & -0.04 & $>5.5$ \\
\hline GL 989 (2) ..... & $1.7(-21)$ & 10.7 & -0.07 & -0.01 & 22. \\
\hline S106/IRS $3 \ldots \ldots \ldots \ldots$ & $1.8(-19)$ & 5.3 & -0.29 & -0.06 & $>3$ \\
\hline 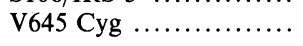 & $1.7(-20)$ & 13.3 & -0.12 & -0.07 & $>1$ \\
\hline MWC $349 \ldots \ldots \ldots \ldots$ & $1.5(-17)$ & 13.0 & 0.03 & 0.06 & 1. \\
\hline$\phi \operatorname{Per} \ldots \ldots \ldots \ldots \ldots \ldots$ & .. & 3.0 & 0.16 & -0.03 & $\cdots$ \\
\hline Typical error...$\ldots \ldots \ldots$ & $\ldots$ & \pm 1 & \pm 0.15 & \pm 0.15 & $\cdots$ \\
\hline Optically thin ........... & $\ldots$ & $\ldots$ & -0.95 & -0.26 & $\ldots$ \\
\hline
\end{tabular}

Note.-Units for $I(\lambda 8542)$ are $\mathrm{W} \mathrm{cm}^{-2}$; parentheses give the power of 10 . Lower limits are $3 \sigma$. All the line fluxes and ratios have been corrected for extinction, except for GL 989 (1) and (2). The $I(\lambda 8662) / I(\lambda 8542)$ ratio does not appear reasonable for GL 437S. Case B theory for underlying Paschen lines gives 0.23 for the $\log$ of the ratio. The $\phi$ Per data are from Polidan (1980).

deblended and reddening corrected line ratios and the equivalent width $W_{\lambda}$ of the $\lambda 8542$ line for each object. Despite the uncertainties due to photon statistics and the deblending scheme, Table 5 shows that the three $\mathrm{Ca}$ II lines are essentially equal in strength. The median values of the line ratios are $1: 1.2: 1.2$ for $\lambda 8498: \lambda 8542: \lambda 8662$, a result that does not depend strongly on the corrections for the underlying Paschen lines. For comparison, the line strengths for optically thin emission should be in the ratio 1:9:5. Equality of $\mathrm{Ca}$ II line strengths has been found in other objects in all cases in which analysis has been done, or where image-tube spectra show the lines clearly. As will be made clear below, certain physical conditions are necessary for the excitation and visibility of the $\mathrm{Ca}$ II lines. It is likely that the various objects displaying the lines strongly, and in equal strength, have regions in which these physical conditions are similar. This must also apply to the embedded sources.

Polidan (1980) and Herbig and Soderblom (1980) have inferred high optical depths in the $\mathrm{Ca}$ II triplet lines from the near equality of the three line strengths in Be stars and T Tauri stars, respectively. The same would appear to be true for the embedded sources, but the situation may be more complicated if the lines are significantly self-reversed, due, for example, to temperature gradients in the emitting volume. The Be star Ca II lines are nearly always self-reversed, while the $\mathrm{H}$ I lines can be either sharply peaked or self-reversed.

The metastable lower levels of the triplet lines decay radiatively in forbidden transitions. These forbidden lines, at $\lambda \lambda 7291,7324$ are suppressed at high electron density, and so their presence in an observed spectrum is indicative of low density $\left(N_{e}<10^{7} \mathrm{~cm}^{-3}\right)$ in the Ca II emitting volume. The spectra were inspected at the position of the $\lambda 7291$ line and upper limits established (see Table 5 ; the $\lambda 7324$ line would in any case be blended with any [O II] $\lambda \lambda 7320,7330$ emission that might be present). The absence of [Ca II] $\lambda 7291$ emission in all objects except MWC 349 and GL 989(2) prevents an unam- biguous determination from being made of the densities in the $\mathrm{Ca}$ II emitting regions. The significances of the upper limits to the $\lambda 7291$ line fluxes presented in Table 5 can only be determined from detailed models of the emitting regions because it is likely that the triplet lines are optically thick (see below).

Because the young sources in our sample have the bolometric luminosities of early B stars, and because strong $\mathrm{H}$ I line emission is present in many of them, it is natural to compare the $\mathrm{Ca}$ II triplet emission characteristics of the embedded sources to those seen in Be stars. The $W_{\lambda}$ 's of the $\lambda 8542$ line in the Be stars of Polidan's (1980) sample display a wide range from one star to another, with a maximum value of about $5 \AA$. For comparison, GL 989 has a $W_{\lambda}$ of $20 \AA$, and several of the other sources have values near $10 \AA$. Note that the present uncertainty concerning the origin of the $8500 \AA$ continuum (see $\S$ III $b$ above) should not seriously affect the $W_{\lambda}$ 's; scattering of the radiation from a hidden source ought to maintain the $W_{\lambda}$. The line fluxes, on the other hand, cannot be interpreted unambiguously if most of the light we see at $8500 \AA$ is scattered. The difference in $W_{\lambda}$ indicates that the $\mathrm{Ca}$ II triplet emission from the embedded sources can be stronger than in Be stars by factors of 2 or 3 .

\section{DISCUSSION}

\section{a) Comparison with Be Stars and T Tauri Stars}

In describing the spectra, we have so far avoided mention of detailed models for the regions in which the lines are formed. Rather, we have emphasized similarities to known and better studied objects which share some of the spectral characteristics of the embedded objects. Indeed, one of the major points of this paper is the realization that the compact embedded sources, previously called protostars, are not the exotic objects they were first imagined to be. Although heavily dust embedded, their immediate circumstellar regions appear now to be quite similar to the circumstellar regions around many other well- 
known classes of visible young emission line stars. The emission line spectra from the compact embedded sources and from these visible young stars originate in this circumstellar region.

There are many similarities between the embedded source spectra and those of other objects, the Be stars in particular. The physical conditions in at least some parts of the envelopes surrounding these classes of objects must therefore be broadly similar. To summarize the main similarities, the Paschen lines have decrements similar to those in $\phi$ Per, O I 28446 emission is present, the $\mathrm{Ca}$ II triplet lines are present, the blended lines near $\lambda 9229$ and the $\lambda 9994$ line are present, the bolometric luminosities are similar to those of Be stars, and the Paschen jump can be in emission. Furthermore, it is interesting that disk geometries are required for the circumstellar envelopes around Be stars and they are also inferred for the embedded sources from the bipolar nature of the molecular outflows detected around these objects, although the sizes of the disks in each case may differ greatly. There is at least one difference between the embedded sources and the Be stars, namely, that of the $\mathrm{H}$ I line widths: the $\mathrm{H}$ I line widths in $\mathrm{Be}$ stars tend to be a factor of 2 wider than in the embedded sources. However, this comparison (also mentioned by Thompson 1982) may be faulty because it refers to the $\mathrm{H} \alpha$ line in Be stars and to Brackett lines in the embedded objects. The embedded source spectra also show certain similarities to the far-red spectra of peculiar emission-line stars (see, e.g., Andrillat and Swings 1976), but not enough quantitative information on the line strengths and line widths for stars in this heterogeneous class of objects is available to allow us to draw firm comparisons. The similarities with Herbig $\mathrm{Ae}-\mathrm{Be}$ stars are also marked. In fact, MWC 297 was included in Herbig's original list of such stars.

Spectroscopic similarities are also evident between the compact embedded sources and the $\mathrm{T}$ Tauri stars. Both are young stellar objects, but the T Tauri stars have lower masses. Again, the hydrogen lines in both classes of objects are in emission and can have widths of several hundred $\mathrm{km} \mathrm{s}^{-1}$, the line decrements deviate from normal Case B recombination, $\mathrm{O}_{\mathrm{I}} \lambda 8446$ emission is often present, and the $\mathrm{Ca}$ II triplet lines are often seen in emission with the three lines having equal strengths. The major differences between the embedded sources and the $T$ Tauri stars are that the embedded sources are more luminous than the T Tauri stars by $\sim 2$ to 3 orders of magnitude, and the photospheric spectra of the T Tauri stars are of later type. Although some of the emission features in $\mathrm{T}$ Tauri stars have sometimes been interpreted as due to surface phenomena such as stellar flares (Herbig and Soderblom 1980), they can equally well be interpreted as circumstellar phenomena, and the similarities with the embedded sources then indicate the existence of circumstellar regions with similar physical conditions around both classes of objects. That the circumstellar phenomena around young stellar objects are independent of the stellar mass is also suggested by the presence of energetic bipolar molecular outflows around many embedded sources and a few T Tauri stars (Edwards and Snell 1983).

It is well known that both T Tauri stars and Be stars display temporal variations in their line strengths and shapes. It will be of interest to monitor the spectra of the embedded sources over time scales of several years to look for analogous changes.

\section{b) Physical Conditions}

We now discuss the physical conditions and line formation mechanisms responsible for the observed spectral features in the embedded sources. A detailed understanding of the region also requires information on the gas motions, information that we plan to discuss in future papers.

$$
\text { i) The Hydrogen Lines }
$$

From the distribution of Paschen line points in Figure 3, it is clear that the hydrogen line ratios in the embedded sources are not consistent with formation under conditions appropriate to the Case B recombination approximation. The Br $\alpha, \mathrm{Br} \gamma$, and Pf $\gamma$ lines confirm this result, where published data of sufficient quality are available. This finding is clearly crucial to a resolution of the line excitation problem; it is not surprising that the hydrogen line fluxes should exceed those predicted for Case $\mathrm{B}$ recombination in a compact $\mathrm{H}$ II region powered by a ZAMS star with the measured luminosity.

Two ideas have been advanced for the hydrogen line excitation in the embedded objects: (1) that a strongly trapped Ly $\alpha$ radiation field overpopulates the hydrogen $n=2$ level, leading to ionizations and further recombinations by Balmer continuum and Lyman line radiation (Simon et al. 1981; Thompson 1984), and (2) that the hydrogen level populations are thermalized and the lines are excited collisionally (Krolik and Smith 1981). Simon et al. (1983) have considered their original model in more detail; the basic idea is that of radiatively ionized wind in which the line photons arise originally from recombination cascades but can be scattered many times before leaving the envelope.

The Paschen line strengths relative to that of $\mathrm{P} 7$, corrected approximately for reddening using the $A_{V}{ }^{\prime}$ values from Table 4, are collected in Table 6 . The lines from P7 to P14 are roughly equal in strength; i.e., they are within a factor of 2 of each other, except in the cases of $\mathrm{LkH} \alpha 101$ and MWC 349. This fact allows us to make a general statement about $\mathrm{H}$ line excitation in the embedded objects. Because the $f$-values of the transitions vary by a factor of 12 from P7 to P14, no line formation mechanism that predicts line strengths which depend strongly

TABLE 6

DeReddened Paschen Line Ratios

\begin{tabular}{|c|c|c|c|c|c|c|}
\hline $\begin{array}{l}\text { Source } \\
\text { (1) }\end{array}$ & $\begin{array}{l}\mathrm{P8} \\
\mathrm{P7} \\
(2)\end{array}$ & $\begin{array}{l}\mathrm{P9} \\
\mathrm{P7} \\
(3)\end{array}$ & $\begin{array}{l}\frac{P 10}{P 7} \\
(4)\end{array}$ & $\begin{array}{l}\mathrm{P} 11 \\
\mathrm{P} 7 \\
(5)\end{array}$ & $\begin{array}{l}\frac{P 12}{P 7} \\
(6)\end{array}$ & $\begin{array}{l}\mathrm{P} 14 \\
\mathrm{P} 7 \\
(7)\end{array}$ \\
\hline IC $1848 \mathrm{~A}$ W(1) ... & 1.4 & $2.6^{\mathrm{a}}$ & 0.5 : & & & 0.6 \\
\hline IC $1848 \mathrm{~A}$ W(2) & 1.3 & $2.5^{\mathrm{a}}$ & 0.6 & 0.8 & 0.8 & 0.6 \\
\hline 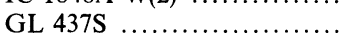 & 2.8 & 3.4 & 1.3 & 1.5 & 2.0 & 1.2 \\
\hline GL $490 \ldots \ldots \ldots$ & 1.4 & $3.0^{\mathrm{a}}$ & 0.7 & 0.9 & 0.9 & 0.6 \\
\hline $\mathrm{LkH} \alpha 101 \ldots \ldots \ldots \ldots \ldots \ldots$ & 1.6 & 1.3 & 0.5 & 0.5 & 0.4 & 0.2 \\
\hline S235B ... & 0.8 & 1.0 & 0.4 & 0.6 & 0.7 & 0.4 \\
\hline GL $961 \ldots$ & 1.1: & 1.0: & & & & 8.0: \\
\hline MWC 297 & 1.1 & 1.2 & 0.6 & 0.6 & 0.6 & 0.5 \\
\hline S106/IRS $3 \ldots \ldots \ldots \ldots \ldots$ & $3.7^{\mathrm{a}}$ & $1.8^{\mathrm{a}}$ & 0.5 & 0.6 & 0.5 & 0.5 \\
\hline V645 Cyg .................. & 1.1 & 2.1 & 0.5 & 0.7 & 0.7 & 0.5 \\
\hline MWC 349 & 2.3 & 1.3 & 0.5 & 0.4 & 0.3 & 0.2 \\
\hline$\phi$ Per $\ldots \ldots \ldots \ldots \ldots \ldots \ldots \ldots \ldots \ldots$ & 0.93 & 0.84 & 0.73 & 0.71 & 0.63 & 0.78 \\
\hline Case $\mathrm{B}$ recombination...... & 0.66 & 0.46 & 0.33 & 0.25 & 0.20 & 0.12 \\
\hline Stellar wind model ........... & 0.70 & 0.52 & 0.41 & 0.32 & 0.27 & 0.19 \\
\hline$\%$ Error $\left(A_{V}=5 \mathrm{mag}\right)^{\mathrm{b}} \ldots \ldots$ & 20 & 37 & 51 & 62 & 71 & 85 \\
\hline
\end{tabular}


upon the $f$-values, either implicitly or explicitly, can explain the decrements. The simplest example of a violation of this condition is Case B recombination theory, and indeed no model can apply in which the line emission is optically thin, no matter what the mechanism for exciting the levels is.

A more complex situation arises in the thermally excited spherically symmetric wind model advanced by Krolik and Smith (1981). The basic idea of this model (which is also discussed by Simon et al. 1983) is that in a spherically symmetric neutral outflowing wind in which the line transitions are optically thick somewhere in the flow, the line fluxes are determined by the effective size of the radiating surface at each frequency in the line. The effective size of the optically thick surface depends on how far into the wind we "see," i.e., where the optical depth in the line is of order unity. This depth clearly depends on the $f$-value of the transition. If the optical depth is small, we see radiation coming predominantly from deep inside the wind, while for optically thick lines the radiation comes mostly from the periphery. It is the size of the region at each wavelength that is the dominant factor in determining line fluxes. Thus the $f$-values of the transitions enter the line ratios through the assumed geometry. To quantify the dependence in the case of the Paschen lines, we note that in the Krolik and Smith model the luminosity of a particular line, $L$, depends on the size of the region, $R$, as follows:

$$
L \propto R^{2} B_{v}\left[T_{\mathrm{ex}}(R)\right] v W,
$$

where the line excitation temperature, $T_{\text {ex }}$, can be a function of $R$, and $W$ is a factor which takes account of deviations of the area of the radiating surface from $4 \pi R^{2}$ due to the particular radial dependences of $T_{\mathrm{ex}}$ and to the velocity gradients which in part govern the line trapping. The size of the region depends on the $f$-value of the line as follows:

$$
R \propto(f / v)^{1 / 3} F\left(T_{\mathrm{ex}}, n\right),
$$

where $F$ is a collection of terms which depend only on $T_{\mathrm{ex}}$ and $n$, the lower level of the transition, for a given model. For the Paschen series, $n$ is constant; and we assume that $F$ is also constant for all lines. The line flux then depends on the $f$-value as

$$
L \propto f^{2 / 3} v^{1 / 3} B_{v}\left[T_{\mathrm{ex}}(R)\right] W .
$$

According to Krolik and Smith, $W$ should be a slowly varying function of order unity for lines on the Rayleigh-Jeans part of the Planck function; $W$ should not vary strongly for the Paschen lines P7 to P14 which are close together in frequency.

Taking specific examples, we list in Table 6 the predictions for the Krolik and Smith model for the case that $W$ is constant. The wind model predictions for P8 through P14 deviate systematically from the observed line ratios, with the greatest discrepancy occurring for $\mathrm{P} 14$ where the model predicts a ratio of 0.2 , while for most of the objects the observed ratio is close to 0.5 . We are inclined to believe that the problem lies with the most strongly varying parameter in the theory, namely the $f$-values, through the assumption of spherical symmetry. A simple alternative is a slab or disk, where the geometry enters less strongly; but other geometries, such as a thin shell, are also possible. We note finally, that preferential scattering of the higher Paschen lines cannot explain the decrements, the ratio of the P7 and P14 frequencies being only 1.17. We have not extended the comparison to the infrared lines because of the uncertainties in the reddening values. ii) The $\mathrm{O}$ I $\lambda 8446$ Line

Morgan (1971) showed that if $\operatorname{Ly} \beta$ fluorescence excites O I $\lambda 8446$, and if $\mathrm{H} \alpha$ is optically thin, then the $\lambda 8446 / \mathrm{H} \alpha$ ratio should be less than $4 \times 10^{-5}$, which is far smaller than the values of $0.01-0.06$ found for the embedded sources. Such large values indicate that the region emitting the $\mathrm{O}$ I line is very optically thick in the $\mathrm{H} \alpha$ line, $\left(\tau \sim 10^{3}\right)$, in order that Ly $\beta$ photons be recycled sufficiently many times to allow their eventual absorption by an $\mathrm{O}$ I atom in the ground state. The requirement that $\mathrm{H} \alpha$ be optically thick has been discussed by Oke and Shields (1976), Netzer and Penston (1976), and Grandi (1980). High optical depth in $\mathrm{H} \alpha$ certainly comes as no surprise in those sources with optically thick Paschen lines.

The $O$ I line excitation mechanism requires that $O$ I be mostly neutral, and owing to efficient charge-exchange reactions between $\mathrm{O} \mathrm{I}$ and $\mathrm{H}$, this requires that $\mathrm{H}$ also be mostly neutral in the $\mathrm{O}_{\mathrm{I}}$ emitting region. Either the $\mathrm{O}_{\mathrm{I}}$ line is excited in transition regions where hydrogen is partially ionized, or both lines are excited in predominantly neutral regions. A specific model of the latter mechanism was developed by Strittmatter et al. (1977) to explain an $\mathrm{O}_{\mathrm{I}} \lambda 8446 / \mathrm{H} \alpha$ ratio of 0.3 in Nova Cygni 1975. The Ly $\beta$ flux required to excite O I fluorescence is also able to ionize $\mathrm{Ca}$ II. Thus either the $\mathrm{Ca}$ II and $\mathrm{O}$ I emitting volumes are physically separated, or a means of shielding $\mathrm{Ca}$ II from this radiation is required.

Although detailed ionization and radiative transfer models are required to fully understand the $\mathrm{O}_{\text {I results, the } \mathrm{H} \alpha \text { optical }}$ depths implied lend support to the idea that ionizations from the $n=2$ level of hydrogen are the solution to Thompson's line excitation problem. For $\tau_{\mathrm{H} \alpha}=10^{3}, \tau$ at the Balmer edge is $\sim 0.1$; this is within range of allowing substantial absorption of the primary Balmer continuum emission of the central star (Thompson 1984; Simon et al. 1983).

iii) The Ca II Lines

In order that the $\mathrm{Ca}$ II triplet lines be seen, the $\mathrm{Ca}$ II emitting region must have physical conditions that are very different from those encountered in the study of compact $\mathrm{H}$ II regions. Because calcium is not very abundant, most of the Ca II emission must arise in regions where most of the calcium is singly ionized. This requires that the electron temperature in the $\mathrm{Ca}$ II emitting region be less than $\sim 5000 \mathrm{~K}$, if the lines are formed in LTE and the electron densities are less than $\sim 10^{12} \mathrm{~cm}^{-3}$ (this condition is easily found from the Saha equation). Under these conditions, hydrogen is predominantly neutral. Thus it is highly unlikely that the $\mathrm{C}$ II triplet lines are emitted from within a region where hydrogen is photoionized. Furthermore, because the ionization potential of $\mathrm{Ca}^{+}$is only $11.8 \mathrm{eV}$, the $\mathrm{Ca}^{+}$ions outside any $\mathrm{H}$ II region must also be shielded from the UV radiation from the central object in the $11.8-13.8 \mathrm{eV}$ range. As discussed by Polidan (1980), it is the $\mathrm{C}_{\mathrm{I}}$ opacity that provides most of the shielding of the $\mathrm{Ca}^{+}$ions from these ionizing photons in Be stars. Firm estimates of the size of the $\mathrm{Ca}$ II emitting volume in the embedded sources are difficult to make, given our incomplete knowledge of the temperature, radiation field, and density in the region. Nevetheless, it is safe to conclude that the $\mathrm{Ca}$ II triplet line emitting region must be significantly removed, either geometrically or in an optical depth sense, from the central object. Polidan (1980) found that for a variety of geometries and physical conditions in the $\mathrm{Ca}$ II emitting zones of Be stars, the size of the zone must be several hundred solar radii, if the stellar surface temperature is $\sim 25,000 \mathrm{~K}$. 
The existence of warm neutral material very close to the central source places strong constraints on the geometrical distribution of this material about the embedded objects. In S106 in particular, a well-developed $\mathrm{H}$ II region exists far outside the core source, and as Lyman continuum photons must be able to penetrate to the $\mathrm{H}$ il region, the neutral material must either be in the form of a disk which is close to the central star or be strongly clumped with a small filling factor. Such a disk would not be on the same spatial scale as the disk envisaged by Bally and Scoville (1982), but rather would extend in to at least the vicinity of the $\mathrm{Ca}$ II emitting region, i.e., only a few hundred solar radii from the central star. How this material could remain neutral during the time it takes for the $\mathbf{H}$ II region in S106 to develop is unclear, but we note that, although they are not well understood (Marlborough 1976; Poeckert 1982), the neutral zones within the envelopes of Be stars have found a way to survive in a similarly harsh environment. In the case of the embedded sources, the envelope geometries must hold important information on whether the collimation of the largescale bipolar outflows is due to the envelope itself (Königl 1982), or is the result of some intrinsic property of the central star (Draine 1983).

Other emission from a region such as is required here may already have been detected in the BN source. Scoville et al. $(1979,1983)$ detected emission at the $2.3 \mu \mathrm{m}$ CO bandheads in this object. The vibrational temperature of the emission is in the range $3000-5000 \mathrm{~K}$ and densities of $n_{\mathrm{H}}>10^{10} \mathrm{~cm}^{-3}$ are inferred. The emission arises close to the central star $(R<300$ $\left.R_{\odot}\right)$. The hot CO gas temperature is similar to that required for the Ca II emitting region. (The density probably is too, but is difficult to determine from the present data.) Scoville et al. (1983) suggested that the similar sizes of the $\mathrm{CO}$ and $\mathrm{H}$ I emitting volumes in $\mathrm{BN}$ are evidence for a disk geometry around this source. However, if the $\mathrm{H} \mathrm{I}$ emission does not originate in a hot $\mathrm{H}$ il region, the disk geometry required to separate the $\mathrm{CO}$ and $\mathrm{H}$ I emitting regions may no longer be required. The $\mathrm{CO}$ lines in $\mathrm{BN}$ are so heavily blended that no useful line widths have so far been determined for them.

\section{iv) Comparison with Active Galaxy Broad-Line Region Clouds}

The physical conditions in the warm neutral medium which we find necessary to explain the line excitation in young stellar objects (YSOs) are reminiscent of those implicit in certain models for the broad-line region in Seyfert 1 galaxies and QSOs. In the models of Weisheit, Shields, and Tarter (1981, hereafter WST) and Grandi (1980), the $n=2$ level of $\mathrm{H}_{\mathrm{I}}$ is populated by a diffuse field of $\operatorname{Ly} \alpha$ radiation which is itself maintained by ionizations which result from the absorption of soft X-rays. These X-ray photons penetrate the neutral medium beyond the $\mathrm{H}$ I Strömgren sphere, and deposit their energy in the "neutral" medium, which is maintained at an ionization level around $20 \%$. The electron temperature in the WST model is around $10^{4} \mathrm{~K}$, which is too hot to allow any Ca II emission, and indeed none is seen. The high $\mathrm{H} \alpha$ optical depth through the medium results from the trapped Ly $\alpha$ photons. Collisions out of $n=2$ to higher levels of hydrogen arise easily if $T \gtrsim 5000 \mathrm{~K}$, and most of the $\mathrm{H}$ I line radiation arises in this way. Some Ly $\beta$ photons are created by recombinations, but most arise from collisions from $n=2$ to $n=3$ with subsequent decay as $\mathrm{Ly} \beta$ as well as $\mathrm{H} \alpha$ and $\mathrm{Ly} \alpha$ radiation. The $\mathrm{Ly} \beta$ photons then excite $\mathrm{O}$ I.

We note that the WST models for the broad line region in active galactic nuclei have densities and column densities very similar to the Be star envelopes to which we have compared the embedded-source emission-line regions. In the WST models, an X-ray photon flux of $10^{16} \mathrm{~cm}^{-2} \mathrm{~s}^{-1}$ at the front surface of a broad line region cloud is needed to heat and partially ionize the medium. This X-ray requirement is not particularly high; at $100 R_{\odot}$ from a normal Be star the coronal $2-6 \mathrm{keV}$ photon flux is $\sim 10^{15} \mathrm{~cm}^{-2} \mathrm{~s}^{-1}$ (Underhill and Doazan 1982). It therefore seems plausible that the X-ray heating mechanism in the embedded sources need not be more than 10 times higher. To our knowledge, no known X-ray source is coincident with a compact embedded object, although detailed searches have not been made yet. Many T Tauri stars are well documented X-ray sources (Feigelson and DeCampli 1981), although the X-ray emission mechanism may be different from that in hotter stars.

We note that the anomalous $\mathrm{Ly} \alpha / \mathrm{H} \alpha$ ratios in QSOs and Seyfert 1 galaxies have been satisfactorily explained in the above quoted papers as an excess of $\mathrm{H} \alpha$ photons, due to the near thermalization of the higher $\mathrm{H}_{\mathrm{I}}$ levels, with the energy in the lines arising ultimately from the X-ray input. We therefore share Krolik and Smith's view that Thompson's line excitation problem is closely related to the $\mathrm{Ly} \alpha / \mathrm{H} \alpha$ problem in QSOs.

\section{SUMMARY}

From CCD spectrophotometry of 15 young stellar objects deeply embedded in molecular clouds we have found the following observational results and basic conclusions. Not all of the results apply to all of the objects; rather, an "average" YSO is described.

1. The overall spectral features resemble those of Be stars (except for the amount of reddening), including several weaker low-excitation lines. Thus the physical conditions in the envelopes of the YSOs are probably similar to those of more easily accessible (visible) objects.

2. A red continuum, probably due to some combination of the flux of the central excitation star and a circumstellar shell, is seen. The continuum data are in some cases consistent with the total source luminosities. However, for some objects scattering of the line and continuum radiation near the central object is probable; this compromises such comparisons.

3. The Paschen lines of $\mathrm{H} \mathrm{I}$ are in emission and are seen to at least P14. The H I Paschen line decrements are not consistent with Case $\mathrm{B}$ recombination theory. The line ratios severely limit models in which the line $f$-values enter expressions for the line strengths strongly. Not only are the lines optically thick, but certain geometries, such as spherically symmetric outflows in which the line ratios are determined by the effective size of the optically thick region, are mitigated against.

4. The $\mathrm{O}_{\mathrm{I}} \lambda 8446$ line is present and excited by $\operatorname{Ly} \beta$ fluorescence, and $\mathrm{H} \alpha$ must have an optical depth of order $10^{3}$. This is qualitatively consistent with the Paschen line optical depths. The solution to Thompson's line excitation problem probably involves ionizations from $n=2$ of hydrogen; the order of magnitude of the $\mathrm{H} \alpha$ optical depth in the stellar envelope implies a Balmer continuum optical depth that is not far from that required for ionization by primary stellar photons in the Balmer continuum.

5. The Ca II triplet is seen strongly in emission, with the three lines roughly equal in strength. The $\mathrm{Ca}$ II emission-line region is also probably optically thick, but it is not possible to derive column densities from the present data. In order that the calcium be mostly singly ionized, the temperature in the $\mathrm{Ca}$ II region must not exceed $5000 \mathrm{~K}$ for densities less than $10^{14}$ $\mathrm{cm}^{-3}$. 
This work was supported by the National Science Foundation. We are especially grateful to Bev Oke and Jim Gunn for providing the excellent instrumentation used to make the observations, and to Todd Boroson whose software expertise and patience seem equally boundless. We also thank Jay Elias, Bev Oke, and Carol Lonsdale for reading the manuscript. We appreciate a careful reading of the paper by an anonymous referee.

\section{APPENDIX}

\section{NOTES ON INDIVIDUAL OBJECTS}

For each object, information on the observational techniques, details of the spectrum, and other data are given. Table 7 lists the exposure times, and references to the photometric and spectroscopic data for the infrared continua and lines.

\section{a) $\$ 106$}

The exciting star, S106/IRS 3, was easily located on the Quantex screen. The seeing was less than 1", and our Quantex integration showed considerably more detail than on published charts. The instrument was rotated to put the slit parallel to the atmospheric dispersion, which was also parallel to the bright southern nebular rim. A $2^{\prime \prime}$ wide slit was used to reduce the emission from the bright nearby nebulosity and a 4 " slit spectrum was also taken to measure the loss of continuum flux due to spillover. A correction of $20 \%$ was applied to the line fluxes in Table 1.

Measurement of the $\mathrm{H} \alpha$ and [S III] $\lambda \lambda 9069,9532$ line fluxes along the slit showed that these lines are spatially extended in the vicinity of IRS 3. This is not obviously the case for any other lines in the spectrum. For $\mathrm{H} \alpha$, approximately half of the flux is from the foreground (nebular) emission, but $\mathrm{H} \alpha$ is definitely detected in IRS 3 . In the case of the $\lambda 9532$ line, the [S III] emission peaks at the location of IRS 3, but is asymmetrically distributed in its vicinity. Other weaker lines in the S106/IRS 3 spectrum which are probably due to nebular emission are He I $\lambda 6678, \mathrm{He}$ I $\lambda 7065$, [Ar III $] \lambda 7135, \mathrm{He}$ I $\lambda 7280$, [O II] $\lambda \lambda 7320$, 7330 , and two unidentified lines at $\lambda 7234$ and $\lambda 7254$ which are seen in the Orion Nebula.

Inspection of the two-dimensional data also showed that at shorter wavelengths a spatially distinct second source of blue light distorts the spectrum. It was not possible to unambiguously separate out this component, so it was included in the final spectrum.

\section{b) IC $1848 A$}

The spectrograph slit was placed across the two visibly bright lobes separated by $\sim 25^{\prime \prime} \mathrm{E}-\mathrm{W}$. The west lobe, which is discussed in this paper, has considerable structure, with a bright condensation to the east and a more diffuse region west. These are referred to as IC $1848 \mathrm{~A} \mathrm{~W}(1)$ and $\mathrm{W}(2)$ in this paper. On a later observing run, the $2 \mu \mathrm{m}$ continuum source was located to a precision of $\sim 0^{\prime \prime} .5$. It lies 1 . 1 east and 0 . 5 south of the center of the bright condensation and so should have been included in our 4 " wide slit spectrum. The apparent agreement

TABLE 7

OBSERVATIONAL SUMMARY

\begin{tabular}{|c|c|c|c|c|c|}
\hline $\begin{array}{c}\text { Source } \\
\text { (1) }\end{array}$ & $\begin{array}{c}\text { Exposure } \\
\text { (s) } \\
\text { (2) }\end{array}$ & $\begin{array}{c}\text { Chart }^{\mathrm{a}} \\
\text { (3) }\end{array}$ & $\begin{array}{l}\text { IR Continuum } \\
\text { Data }^{\mathrm{a}} \\
\text { (4) }\end{array}$ & $\begin{array}{c}\text { IR H-Line } \\
\text { Data }^{\mathrm{a}} \\
(5)\end{array}$ & $\begin{array}{c}\text { Radio } \\
\text { Data }^{a} \\
(6)\end{array}$ \\
\hline IC $1848 A \ldots$ & 2400 & $1,2,3$ & 17 & $\ldots$ & $\ldots$ \\
\hline GL $437 \ldots \ldots$ & 2400 & 4 & 4 & $\ldots$ & $\ldots$ \\
\hline GL $490 \ldots \ldots \ldots \ldots \ldots \ldots$ & 3600 & 5 & 18 & 24 & 32 \\
\hline $\mathrm{LkH} \alpha 101 \ldots \ldots \ldots \ldots \ldots$ & 600 & 6 & 19 & 25,26 & 33 \\
\hline S235B $\ldots \ldots \ldots \ldots \ldots$ & 2400 & 7 & 20 & 27,28 & 34 \\
\hline GL 961 & 2400 & 8 & 10 & 24 & 35 \\
\hline GL $989 \ldots$ & 2400 & 9 & 10 & $\ldots$ & $\ldots$ \\
\hline M8E $\ldots \ldots \ldots \ldots \ldots \ldots \ldots$ & 3600 & 10 & 10 & $\ldots$ & $\ldots$ \\
\hline M17/IRS $1 \ldots \ldots \ldots \ldots$ & 5400 & 11 & 21 & $\ldots$ & $\ldots$ \\
\hline MWC $297 \ldots \ldots \ldots \ldots$ & 10 & $\ldots$ & 10 & 29 & $\ldots$ \\
\hline S106/IRS $3 \ldots \ldots \ldots \ldots$ & 2400 & 12,13 & 12,22 & 24 & 35 \\
\hline V645 Cyg ................ & 200 & 14 & 23 & $\ldots$ & $\ldots$ \\
\hline MWC $349 \ldots \ldots \ldots \ldots$ & 10 & 15 & 19 & 29 & 36 \\
\hline NGC $7538 /$ IRS $2 \ldots \ldots$. & 2400 & 16 & $\ldots$ & 30,31 & $\ldots$ \\
\hline
\end{tabular}

a REFERENCES.-(1) Thronson et al. 1980. (2) Gosnell, Hudson, and Puetter 1979. (3) Cohen and Lewis 1978. (4) Wynn-Williams et al. 1981. (5) Cohen 1975. (6) Herbig 1971. (7) Glushkov, Denisyuk, and Karagina 1975. (8) Cohen 1973. (9) Allen 1972. (10) Allen et al. 1977. (11) Beetz et al. 1976. (12) Pipher et al. 1976. (13) Eiroa, Elsässer, and Lahulla 1979. (14) Cohen 1977. (15) Herbig and Mendoza 1960. (16) Wynn-Williams, Becklin, and Neugebauer 1974. (17) Loren and Wooten 1978. (18) Merrill and Stein 1976. (19) Allen 1973. (20) Evans and Blair 1981. (21) Kleinmann and Wright 1973. (22) Gehrz et al. 1982. (23) Humphreys, Merrill, and Black 1980. (24) Simon et al. 1983. (25) Thompson et al. 1977. (26) Simon, Simon, and Joyce 1979. (27) Tokunaga and Thompson 1979. (28) Krassner et al. 1982. (29) Thompson et al. 1977. (30) Werner et al. 1979. (31) Fischer et al. 1980. (32) Simon et al. 1981. (33) Cohen, Bieging, and Schwartz 1982. (34) Israel and Felli 1978. (35) Bally and Predmore 1983. (36) Olnon 1975. 
between the near-infrared photometric data and our new data seems somewhat fortuitous considering the complex structure of the source and the size of the slit relative to it.

The spectrum of IC 1848A East is not discussed in this paper. It displays the Paschen lines in absorption, and $\mathrm{O}_{\mathrm{I}}$ $\lambda 8446$ and $\mathrm{H} \alpha$ in emission. The spectrum is presumably light reflected from a central star (Cohen and Lewis 1978).

\section{c) $L k H \alpha 101$}

This object is so bright that several of the strong lines are saturated. The symmetric $\mathrm{H} \alpha$ and $\mathrm{O}_{\mathrm{I}} \lambda 8446$ line shapes are not real but are caused by smearing during readout of the CCD. There are many fainter lines in the spectrum (see also Herbig 1971) which require higher spectral resolution for detailed study. High-resolution, unsaturated spectra of $\mathrm{H} \alpha$ and the $\mathrm{Ca}$ II triplet region are presented in Persson, McGregor, and Cohen (1984).

\section{d) $G L 437$}

Sources GL 437W and S were visible on the Quantex screen, and both spectra were recorded simultaneously. The 4 " slit was wide enough to include most, but probably not all, of the emission from both sources. This is borne out by the energy distributions in Figure 2. GL $437 \mathrm{~W}$ is a strong radio source, and GL $437 \mathrm{~S}$ is the bright $3 \mu \mathrm{m}$ continuum source discussed by Wynn-Williams et al. (1981). It is interesting that GL 437S shows strong $\mathrm{Ca}$ II and $\mathrm{H}$ I emission but GL $437 \mathrm{~W}$ does not.

Inspection of the two dimensional raw spectra showed that the $\mathrm{H} \alpha$ and $\mathrm{O}_{\mathrm{I}} \lambda 8446$ emission is well localized to each source. There is little or no foreground $\mathrm{H} \alpha$ emission.

\section{e) $G L 490$}

The very red object marked on Cohen's (1975) chart (see his note added in proof) was found with no difficulty. The position of this object does not, however, agree with that listed by Wynn-Williams (1982) and originally by Low et al. (1976). On a subsequent Hale $5 \mathrm{~m}$ observing run the $4 \mu \mathrm{m}$ position of GL 490 was measured; it agrees to within $1^{\prime \prime}$ with the optical position, which is $\alpha(1950)=03^{\mathrm{h}} 23^{\mathrm{m}} 38^{\mathrm{s}} .9, \delta(1950)=58^{\circ} 36^{\prime} 33^{\prime \prime}$. This position agrees well with that of Simon et al. (1983), (although their VLA right ascension position contains a typographical error) and is $19.5 \mathrm{~S}$ and $19^{\prime \prime} \mathrm{W}$ of that listed by Low et al. (1976). There are no neighboring sources or diffuse emission to confuse the spectrum; the $1 \mu \mathrm{m}$ and broad-band pieces of the energy distribution agree well (see Fig. 2).

\section{f) $G L 961$}

The GL 961 continuum source is located 35" east of the easily visible fan-shaped nebula which is referred to here as GL 961 FAN. Although the infrared source was not visible on the Quantex screen, the source was clearly detected in the 0.6-1.0 $\mu \mathrm{m}$ region. Figure 2 shows that the slit may not have included all the flux from the embedded object, but the agreement of the $1 \mu \mathrm{m}$ spectrum of GL 961 with near-infrared photometry is reasonable. Although line fluxes derived from the GL 961 FAN spectrum are not meaningful due to the extended nature of the source, the line ratios can be used to discuss the physical conditions in the region. The $\mathrm{Ca}$ II triplet region in the GL $961 \mathrm{FAN}$ spectrum is confused. The $\mathrm{Ca}$ II lines are seen in emission, but the ratios may be affected by the blended Paschen lines which appear to be in absorption. Consequently this spectrum is not considered in Table 5.

\section{g) GL 989}

The infrared source is reported to lie near the apex of a bright fan-shaped nebula. The instrument was rotated to place the slit along the axis of the fan, through a bright condensation near its tip. No other very red source was visible, and the position in Wynn-Williams's (1982) compilation was not precise enough to allow a blind offset. Two spectra are discussed in this paper. GL 989 (1) is the bright and easily visible starlike condensation near the tip of the fan. GL 989 (2) is an integration of $14^{\prime \prime}$ along the slit in the diffuse fan, uncontaminated by emission from GL 989 (1). The spectrum of the bright condensation [GL 989 (1) in this paper] is clearly not that of the infrared source, as is evident in the composite energy distribution in Figure 2. It is probable that much of the light from the infrared source is reflected in the fan. The $\mathrm{Ca}$ II triplet lines are extended across the entire fan nebula. Because they may be optically thick, it seems certain that they are reflected light. Comparisons of the radio emission and of the infrared $\mathrm{H}$ I line fluxes from GL 989 with the reflected light we measure is not meaningful and has not been included in Figure 3.

The noise in the GL 989 (1) and (2) spectra has a spiky, negative-going character. This is the result of a problem in the sky subtraction procedure. Because of the extended nature of the source, it was necessary to take the sky position far from the object spectra, and a slight tilt in the spectra with respect to the rows of the CCD resulted in imprecise cancellation of the sky background.

\section{h) V645 Cygni}

Two spectra of V645 Cyg were recorded. In the first, the spectrograph slit was placed across knot NO (i.e., V645 Cyg itself, Cohen 1977) with the slit oriented NE-SW. The second spectrum was recorded with the slit in the same orientation but placed across knot N1, an arc of nebulosity probably reflecting light from NO. The seeing was $\sim 1^{\prime \prime}$, and neither spectrum is contaminated with emission from the other knot. Except for the absolute flux levels, the two spectra are identical, as would be expected for wavelength-independent reflection nebulosity. The Ca II triplet lines are very strong in these spectra.

\section{i) NGC 7538}

Our intention was to place the spectograph slit through both IRS 1 and IRS 2. Two faint objects were visible on the Quantex screen close to the positions of these objects, and it is likely that they are the infrared objects. The $1 \mu$ m flux densities agree well with power law extrapolations to shorter wavelengths of the energy distributions given by Wynn-Williams, Becklin, and Neugebauer (1974), but are not plotted in Figure 2 because of the extended nature of IRS 2 and the low signal-to-noise ratio of the IRS 1 data. The spectrum of NGC 7538/IRS 1 is a faint, featurless continuum; the $1 \mu$ flux density is $1.5 \mathrm{mJy}$. No emission lines could be reliably measured for this source. The spectrum of IRS 2 is notable in having exceedingly bright [S III] lines which are extended on a scale of several arcseconds. The emission at $\mathrm{H} \alpha$ in the vicinity of IRS 2 is also extended on a scale of several arcseconds. The anomalous strength of $\mathrm{H} \alpha$ in our spectrum relative to the Paschen lines is most likely due to foreground emission, although scattering or patchy extinction could also be responsible. Only a few other lines, including the [O II $] \lambda \lambda 7320,7330$ doublet, are detectable in the spectrum.

\section{j) $S 235 B$}

This source was easily visible on the Quantex screen. The $\mathrm{H} \alpha$ line may be extended beyond the continuum source. 


\section{k) $M 17 / \operatorname{IRS} 1$}

The source was visible on the Quantex screen, but the average airmass of the observations was 1.6 and bad seeing caused some loss of flux from the $4^{\prime \prime}$ slit. The resulting uncer1 t tainty in the continuum level is $\pm 30 \%$. The $\mathrm{H}$ I Paschen series is not apparent in our spectrum (and therefore M17/IRS 1 was omitted from Fig. 3), but a strong emission feature is seen at the wavelength of $\mathrm{H}_{\text {I }} \mathrm{P}$ 9. This feature is probably due to the same blend of lines seen in the other source spectra and in Be stars (see $\S$ III $f$ ). Very weak emission features are seen at the positions of the $\mathrm{Ca}$ II triplet lines, but the noise level precludes a definite detection.

\section{l) $M 8 E$}

After offsetting from a nearby SAO star, a faint nebulous patch could be seen on the Quantex screen close to the published infrared position. The slit was placed through this patch in an E-W orientation. The poor agreement of the $1 \mu \mathrm{m}$ flux density with the near infrared flux distribution (Fig. 2) indicates that the infrared object was probably not seen directly. No $\mathrm{H}$ I Paschen lines are visible in the spectrum, and the source was omitted from Figure 3.

\section{m) $M W C 349$}

The spectrograph slit was rotated to a position angle of $32^{\circ}$ to avoid the fainter blue companion to MWC 349 (Brugel and Wallerstein 1979).

\section{REFERENCES}

Allen, D. A. 1972, Ap. J. (Letters), 172, L55. 1973, M.N.R.A.S., 161, 145 .

Allen, D. A., Hyland, A. R., Longmore, A. J., Caswell, J. L., Goss, W. M., and Haynes, R. F. 1977, Ap. J., 217, 108.

Andrillat, Y., and Swings, J. P. 1976, Ap. J. (Letters), 204, L123.

Bally, J., and Lada, C. J. 1983, Ap. J., 265, 824.

Bally, J., and Predmore, R. 1983, Ap. J., 265, 778.

Bally, J., and Scoville, N. Z. 1982, Ap. J., 255, 497.

Beetz, M., Elsässer, H., Poulakos, C., and Weinberger, R. 1976, Astr. Ap., 50, 41.

Bowen, I. S. 1947, Pub. A.S.P., 59, 196

Brocklehurst M. 1971 M N R A S 153, 471.

Brugel, E. W., and Wallerstein, G. 1979, Ap. J. (Letters), 229, L23.

Ciatti, F., d'Odorico, S., and Mammano, A. 1974, Astr. Ap., 34, 181.

Cohen, M. 1973, Ap. J. (Letters), 185, L75.

$1975, A J \mathbf{8 0} 125$ $1977, A$. $J . \mathbf{2 1 5}, 533$

Cohen, M., Bieging, J. H., and Schwartz, P. R. 1982, Ap. J., 253, 707.

Cohen, M., and Lewis, R. R. 1978, M.N.R.A.S., 184, 801.

Draine, B. T. 1983 , A p. J., 270, 519.

Edwards, S., and Snell, R. L. 1983, Ap. J., 270, 605.

Eiroa, C. Elsässer, H., and Lahulla, J. F. 1979, Astr. Ap. 74, 89.

Evans, N. J., II, and Blair, G. N. 1981, Ap. J., 246, 394.

Feigelson, E. D., and DeCampli, W. M. 1981, Ap. J. (Letters), 243, L89.

Feinstein, A. 1982, in IAU Symposium 98, Be Stars, ed. M. Jaschek and H.-G. Groth (Dordrecht: Reidel), p. 235

Fischer, J., Righini-Cohen, G., Simon, M., Joyce, R. R., and Simon, T. 1980, Ap. J. (Letters), 240, L95.

Garrison, L. M., Jr. 1978, Ap. J., 224, 540.

Gehrz, R. D., Grasdalen, G. L., Castelaz, M., Gullixson, C., Mozurkewich, D. and Hackwell, J. A. 1982, Ap. J., 254, 550.

Gehrz, R. D., Hackwell, J. A., and Jones, T. W. 1974, Ap. J., 191, 675.

Giles, K. 1977, M.N.R.A.S., 180, 57P.

Glushkov, Yu. I., Denisyuk, E. K., and Karyagina, Z. V. 1975, Astr. Ap., 39, 481.

Gosnell, T., Hudson, H., and Puetter, R. 1979, A.J., 84, 538.

Grandi, S. A. 1975, Ap.J., 196, 465.

.1980, Ap. J., 238, 10.

Herbig, G. H. 1971, Ap. J., 169, 537

Herbig, G. H., and Mendoza, E. E. 1960, Bol. Obs. Tonantzintla y Tacubaya, 2 21 .

Herbig, G. H., and Soderblom, D. R. 1980, Ap. J., 242, 628.

Humphreys, R. M., Merrill, K. M., and Black, J. H. 1980, Ap. J. (Letters), 237, L17.

Israel, F. P., and Felli, M. 1978, Astr. Ap., 63, 325.

Johansson, S. 1977, M.N.R.A.S., 178, 17P.

Johnson, H. L. 1968, in Nebulae and Interstellar Matter, ed. B. M. Middlehurs and L. H. Aller (Chicago: University of Chicago Press), p. 167

. 1978, Revista Mexicana Astr. Ap. 4, 3.

Johnson, H. L., Wisniewski, W. Z., and Fäy, T. D. 1978, Revista Mexicana Astr. Ap., 2, 273

Johnson, H. M. 1968, in Nebulae and Interstellar Matter, ed. B. M. Middle-

hurst and L. H. Aller (Chicago: University of Chicago Press), p. 65.

Kleinmann D. E and Wright E. L. 1973, Ap.J.(Letteers), 185, L131.

Königl, A. 1982, Ap. J., 261, 115.

Krassner, J., Pipher, J. L., Sharpless, S., and Herter, T. 1982, Astr. Ap., 109, 223.

Krolik, J. H., and Smith, H. A. 1981, Ap. J., 249, 628.
Kurucz, R. L. 1979, Ap. J. Suppl., 40, 1

Loren, R. B., and Wooten, H. A. 1978, Ap. J. (Letters), 225, L81.

Low, F. J., Kurtz, R. F., Vrba, F. J., and Rieke, G. H. 1976, Ap. J. (Letters), 206, L153.

Marlborough, J. M. 1976, in IAU Symposium 70, Be and Shell Stars, ed. A. Slettebak (Dordrecht: Reidel), p. 335.

Mendoza, E. E. 1982, in IAU Symposium 98, Be Stars, ed. M. Jaschek and H.-G. Groth (Dordrecht: Reidel) p. 3.

Merrill, K. M., and Stein, W. A. 1976, Pub. A.S.P., 88, 874.

Morgan, L. A. 1971, M.N.R.A.S., 153, 393.

Netzer, H., and Penston, M. V. 1976, M.N.R.A.S., 176, 319.

Oke, J. B., and Gunn, J. E. 1982, Pub. A.S.P., 94, 586 1983, Ap.J., 266, 713 .

Oke, J. B., and Shields, G. A. 1976, Ap. J., 207, 713

Olnon, F. M. 1975, Astr. Ap., 39, 217

Panagia, N. 1973, A.J., 78, 929.

Persson, S. E. Geballe, T. R., McGregor, P. J., Lonsdale, C. J, Edwards, S. and Baas, F. 1984, Ap. J., 286, in press.

Persson, S. E., McGregor, P. J., and Cohen, J. G. 1984, Ap. J., in preparation.

Pipher, J. L., Sharpless, S., Savedoff, M. P., Kerridge, S. J., Krassner, J., Schurmann, S., Soifer, B. T., and Merrill, K. M. 1976, Astr. Ap., 51, 255.

Poeckert, R. 1982, in IAU Symposium 98, Be Stars, ed. M. Jaschek and H.-G. Groth (Dordrecht: Reidel), p. 453.

Polidan, R. S. 1976, in IAU Symposium 70, Be and Shell Stars, ed. A. Slettebak (Dordrecht: Reidel), p. 401.

.1980, Ph.D. thesis, UCLA

Polidan, R. S., and Peters, G. J. 1976, in IAU Symposium 70, Be and Shell Stars, ed. A. Slettebak (Dordrecht: Reidel), p. 59

Rank, D. M., Dinerstein, H. L., Lester, D. F., Bregman, J. D., Aitken, D. K., and Jones, B. 1978, M.N.R.A.S. 185, 179.

Scargle, D. S., Erickson, E. F., Witteborn, F. C., and Strecker, D. W. 1978, $A p$. $J ., 224,527$.

Scoville, N. Z., Hall, D. N. B., Kleinmann, S. G., and Ridgway, S. T. 1979, Ap. J.(Letters), 232, L121.

Scoville, N. Z., Hall, D. N. B., Kleinmann, S. G., and Ridgway, S. T. 1982 Henry Draper Symposium, Ann. N.Y. Acad. Sci., 395, 125.

Scoville, N. Z., Kleinmann, S. G., Hall, D. N. B., and Ridgway, S. T. 1983, Ap. $J ., 275,201$.

Simon, M., Felli, M., Cassar, L., Fischer, J., and Massi, M. 1983, Ap. J., 266 , 623.

Simon, M., Righini-Cohen, G., Fischer, J., and Cassar, L. 1981, Ap. J., 251, 552.

Simon, T., Simon, M., and Joyce, R. R. 1979, Ap. J., 230, 127.

Staude, H. J., Lenzen, R., Dyck, H. M., and Schmidt, G. D. 1982, Ap. J., 255, 95

Strittmatter, P. A. et al. 1977, Ap. J., 216, 23.

Swings, J. P., and Klutz, M. 1976, Astr. Ap., 46, 303.

Thompson, R. I. 1981, in IAU Symposium 96, Infrared Astronomy, eds. C. G. Wynn-Williams and D. P. Cruikshank (Dordrecht: Reidel), p. 153. 1982, Ap. J. 257, 171.

1984 preprint.

Thompson, R. I., Strittmatter, P. A., Erickson, E. F., Witteborn, F. C., and Strecker, D. W. 1977, Ap. J., 218, 170 .

Thompson, R. I., Thronson, H. A., and Campbell, B. 1983, Ap. J., 266, 614.

Thronson, H. A., Jr., Thompson, R. I., Harvey, P. M., Rickard, L. J., and Tokunaga, A. T. 1980, Ap.J., 242, 609.

Tokunaga, A. T., and Thompson, R. I. 1979, Ap.J., 233, 127

Underhill, A., and Doazan, V. 1982, in Be Stars with and without Envelopes (NASA pub. SP-456), p. 60. 
Weisheit, J. C., Shields, G. A., and Tarter, C. B. 1981, Ap. J., 245, 406 (WST).

Werner, M. W., Becklin, E. E., Gatley, I., Matthews, K., Neugebauer, G., and

Wynn-Williams, C. G. 1979, M.N.R.A.S., 188, 463 .

! Whitford, A. E. 1958, A.J., 63, 201.

Iol Wynn-Williams, C. G. 1982, Ann. Rev. Astr. Ap., 20, 587.

10I . 1983, in Galactic and Extragalactic Infrared Spectroscopy, 16th

III ESLAB Symposium, ed. M. F. Kessler and J. P. Phillips, p. 131.

Judith G. Cohen : California Institute of Technology, Mail Code 105-24, Pasadena, CA 91125

Peter J. McGregor : Mount Stromlo and Siding Spring Observatories, Private Bag, Woden P.O., ACT 2606, Australia

S. Eric Persson: Mount Wilson and Las Campanas Observatories, 813 Santa Barbara Street, Pasadena, CA $91101-1292$
Wynn-Williams, C. G., Becklin, E. E., Beichman, C. A., Capps, R., Kleinmann, S. G., and Shakeshaft, J. R. 1981, Ap. J., 246, 801

Wynn-Williams, C. G., Becklin, E. E., and Naugebauer, G. 1974, Ap. J., 187, 